\title{
THE 100 Myr STAR FORMATION HISTORY OF NGC 5471 FROM CLUSTER AND RESOLVED STELLAR PHOTOMETRY
}

\author{
Rubén García-Benito ${ }^{1,2}$, Enrique Pérez ${ }^{3}$, Ángeles I. Díaz ${ }^{2}$, Jesús Maíz Apellániz $^{3,4}$, And Miguel Cerviño ${ }^{3}$ \\ ${ }^{1}$ Kavli Institute of Astronomy and Astrophysics, Peking University, 100871 Beijing, China; luwen@ @ku.edu.cn \\ ${ }^{2}$ Departamento de Física Teórica (C-XI), Facultad de Ciencias, Universidad Autónoma de Madrid, \\ Ctra. Colmenar Viejo km 15.600, E-28049 Madrid, Spain; angeles.diaz@uam.es \\ ${ }^{3}$ Instituto de Astrofísica de Andalucía, CSIC, Aptdo. Correos 3004, E-18080 Granada, Spain; eperez@iaa.es, jmaiz@iaa, mcs@iaa.es \\ Received 2010 August 23; accepted 2011 February 1; published 2011 March 8
}

\begin{abstract}
We show that star formation in the giant $\mathrm{H}$ II region NGC 5471 has been ongoing during the past 100 Myr. Using Hubble Space Telescope/Wide-Field Planetary Camera 2 F547M and F675W, ground-based JHK , and GALEX FUV and NUV images, we have conducted a photometric study of the star formation history (SFH) in the massive giant extragalactic H II region NGC 5471 in M101. We perform a photometric study of the color-magnitude diagram (CMD) of the resolved stars and an integrated analysis of the main individual star-forming clusters and of NGC 5471 as a whole. The integrated UV-optical-NIR photometry for the whole region provides two different reference ages, $8 \mathrm{Myr}$ and $60 \mathrm{Myr}$, revealing a complex SFH, clearly confirmed by the CMD-resolved stellar photometry analysis. The spatial distribution of the stars shows that the star formation in NGC 5471 has proceeded along the whole region during, at least, the last $100 \mathrm{Myr}$. The current ionizing clusters are enclosed within a large bubble, which is likely to have been produced by the stars that formed in a major event $\sim 20 \mathrm{Myr}$ ago.
\end{abstract}

Key words: H II regions - Hertzsprung-Russell and C-M diagrams - galaxies: star clusters: individual (NGC 5471)

Online-only material: color figures, machine-readable and VO tables

\section{INTRODUCTION}

Giant extragalactic H II regions (GEHRs) are important star formation tracers in the chemical evolution of galaxies and can be used as standard candles to estimate cosmological distances. Thus, they have been extensively studied. Equally important, they are the best indicators of the conditions that lead to massive star formation. These objects are very luminous $\mathrm{H} \alpha$ emitters. Their $\mathrm{H} \alpha$ luminosities, in the range $10^{39}-10^{41} \mathrm{erg} \mathrm{s}^{-1}$, imply large numbers of ionizing photons, $10^{51}-10^{53}$ photons $^{-1}$, an ionizing power equivalent to up to several thousands of O5V stars. Such a massive stellar concentration provides an excellent laboratory in which to study the modes of massive star formation.

The energy deposited in the interstellar medium in the form of stellar winds, supernova explosions, and ultraviolet light produces complex stellar and gaseous spatial distributions which change rapidly during the few Myr following the onset of the first massive star generation. Although the massive young clusters that power the GEHRs are expected to form on a timescale shorter than $10 \mathrm{Myr}$, some studies in regions such as 30 Doradus in the Large Magellanic Cloud (LMC) reveal that their stars may not be coeval (Walborn et al. 1999). The first population formed in the central regions may have induced star formation in the periphery through the action of UV radiation and stellar winds that compress the surrounding molecular clouds. Therefore, the study of the interrelation between stars, gas, and dust components is highly relevant for understanding the nature of these regions.

30 Doradus in the LMC and NGC 604 in M33 are the two largest GEHRs in the Local Group and they have been the subject of very detailed analyses. Both are dominated by very young (2-4 Myr) ionizing stellar populations, but they present

\footnotetext{
4 Ramón y Cajal Fellow.
}

two rather different stellar distributions. While NGC 604 is an extended Scaled OB Association with 200 O+WR massive stars surrounded by nebular filamentary structures, 30 Doradus is powered by a Super Star Cluster (SSC) - a much more compact core (Maíz Apellániz 2001) — and a halo, with different stellar populations ranging from 2 to $20 \mathrm{Myr}$ (Walborn \& Blades 1997). Both GEHRs include an ongoing star formation with young stars still embedded in their parent molecular cloud. The structural similarities and differences between these wellstudied and spatially well-resolved Local Group GEHRs have made them obvious ideal laboratories in which to study massive star formation, but in order to extrapolate our knowledge to distant luminous unresolved starbursts it is critically necessary to go a step further and to analyze in detail the somewhat more distant GEHRs just outside the neighborhood of the Local Group.

M101 (NGC 5457) is a giant spiral galaxy located at a distance of 7.2 Mpc (Stetson et al. 1998) yielding a linear scale of $34.9 \mathrm{pc} \operatorname{arcsec}^{-1}$. This galaxy contains a large number of very luminous GEHRs, of which NGC $5471\left(\alpha_{2000}=14^{\mathrm{h}} 04^{\mathrm{m}} 29^{\mathrm{s}}\right.$, $\left.\delta_{2000}=+54^{\circ} 23^{\prime} 48^{\prime \prime} ; l=101.78, b=+59^{\circ} .63\right)$ is one of the outermost, at a galactocentric radius of about $25 \mathrm{kpc}$ (see Figure 1). The $\mathrm{H} \alpha$ morphology of NGC 5471 shows multiple cores with surrounding nebular filaments, extending over a diameter of $\sim 17^{\prime \prime}$, or $\sim 600 \mathrm{pc}$. Ground-based photometry shows five bright knots, designated by Skillman (1985) as components A, B, C, D, and E. Component A is as luminous as 30 Doradus, while components B, C, and E are comparable to NGC 604, the two prototypical spatially resolved mini-starbursts in the Local Group. Thus, NGC 5471 is truly a giant star-forming region.

All these features make NGC 5471 an excellent candidate for the study of complex star formation, being close to the limit distance between the spatially well-resolved Local Group GEHRs and the more distant unresolved starbursts, thus providing an 


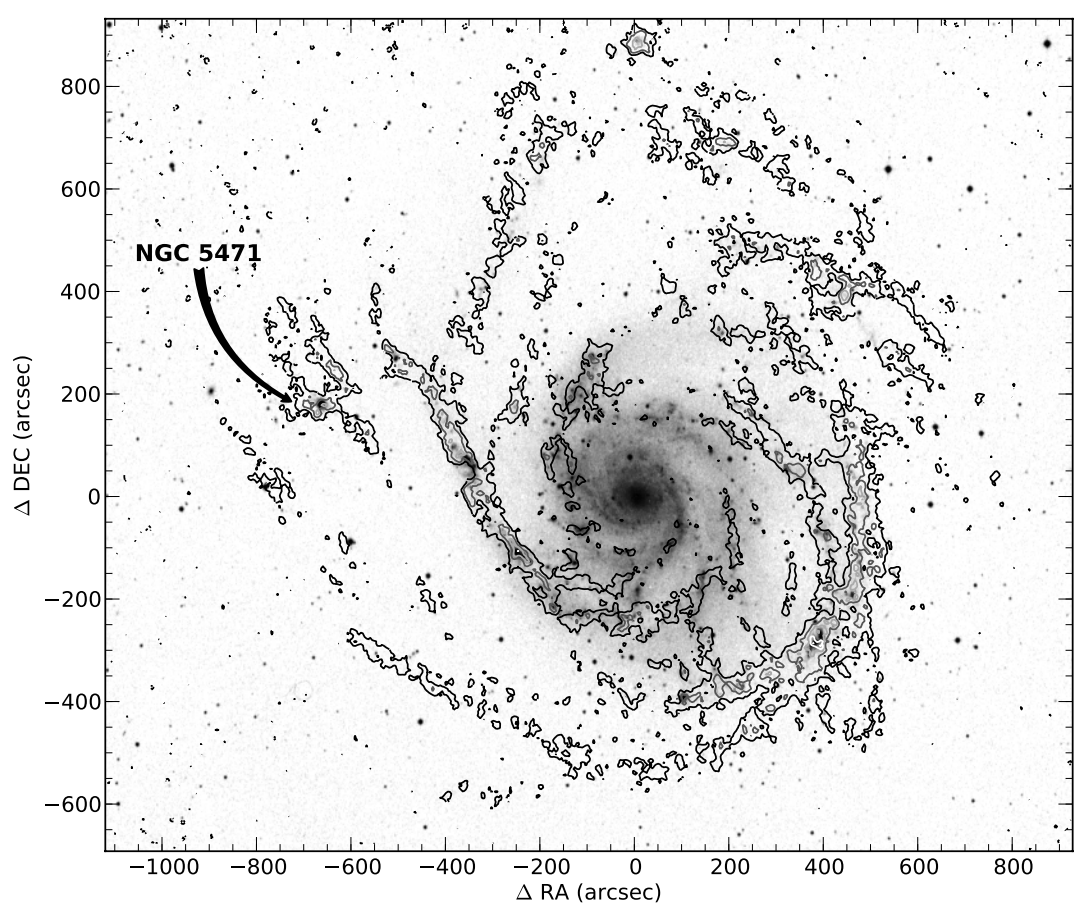

Figure 1. Large-scale image of M101. The gray scale is the Digital Sky Survey image and the contour image is the H I from the VLA (Braun 1995). NGC 5471 is located in the outskirts of the galaxy, in an arm concentration of $\mathrm{H}$.

(A color version of this figure is available in the online journal.)

intermediate step in our understanding of very massive starforming regions.

This paper is organized as follows. Section 2 describes the observations and data reduction. Section 3 presents the integrated analysis of both the individual large clusters and the whole of NGC 5471, and the photometry of the resolved stars. The results found in Section 3 are elaborated and discussed in Section 4. Finally, the conclusions are summarized in Section 5.

\section{OBSERVATIONS AND DATA REDUCTION}

We have retrieved ultraviolet NUV and FUV images of M101 from the GALEX archive as well as broad- and narrowband optical images for NGC 5471 from the Hubble Space Telescope (HST) archive. Finally, we have obtained near-infrared $J H K$ images.

\subsection{GALEX Data}

The GALEX far-ultraviolet (FUV; $\lambda_{\text {ref }}=1530 \AA, \Delta \lambda=$ $400 \AA$ ) and near-ultraviolet (NUV; $\lambda_{\text {ref }}=2310 \AA, \Delta \lambda=$ $1000 \AA$ ) images of M101 were retrieved from the Nearby Galaxies Survey.

The GALEX instrument is described by Martin et al. (2005) and its on-orbit performance by Morrissey et al. (2005). GALEX FUV and NUV imaging was obtained with total exposure times of $1041 \mathrm{~s}$ in each band. The $1 \sigma \mathrm{NUV}$ (FUV) sensitivity limit of the GALEX images is 27.6 (27.5) $\mathrm{AB}$ mag $\operatorname{arcsec}^{-2}$ for these data (Bianchi et al. 2005). At the distance of M101, the linear scale of $34.9 \mathrm{pc} \operatorname{arcsec}^{-1}$ implies that the GALEX point-spread function (PSF) of 4.6 arcsec translates to $160 \mathrm{pc}$; this is barely sufficient to resolve some of the structure in NGC 5471, and we will only use the GALEX data to study the integrated photometry.

\subsection{HST/WFPC2 Imaging}

HST WFPC2 images of NGC 5471 were retrieved from the HST archive. The images were taken on 1997 November
Table 1

Journal of $H S T /$ WFPC2 Observations of NGC 5471

\begin{tabular}{lccc}
\hline \hline Filter & Band & Exposure (s) & Observation ID \\
\hline F547M & Strömgren y & $2 \times 600$ & U4DN030DR, U4DN030ER \\
& & $2 \times 100$ & U4DN030FR, U4DN030GR \\
& & 20 & U4DN030HM \\
F675W & WFPC2 R & $2 \times 400$ & U4DN0308R, U4DN0309R \\
& & $2 \times 50$ & U4DN030AR, U4DN030BR \\
& & 10 & U4DN030CR \\
F656N & H $\alpha$ & 180 & U4DN0305R \\
& & $2 \times 600$ & U4DN0306R, U4DN0307R \\
F673N & {$[\mathrm{S}$ II $]$} & $3 \times 700$ & U4DN0301R, U4DN0302R, U4DN0303R \\
& & 300 & U4DN0304R \\
\hline
\end{tabular}

Notes. All images were obtained on 1997 November 1 for the cycle 6 program GO-6928, with You-Hua Chu as PI. The object of study is centered in the WF3 camera.

1 through two emission-line filters: F656N ( H $\alpha)$ and F673N ([S II]), and two continuum filters: F547M (Strömgren y) and F675W (WFPC2 R; Heyer \& Biretta 2004). Each camera images onto a Loral $800 \times 800$ CCD which gives a plate scale of 0 '.046 $^{\prime}$ pixel $^{-1}$ for the PC camera and $0^{\prime} .10$ pixel $^{-1}$ for the three WF cameras, with a readout noise of $\sim 5 e^{-}$and a gain of $7 e^{-} / \mathrm{DN}$ for this observations. NGC 5471 is centered in the WF3 camera in all images. The scale of the WF CCDs at NGC 5471, for which we assume a distance modulus of $(m-M)=29.3$ (Stetson et al. 1998), is $3.5 \mathrm{pc} \mathrm{pixel}^{-1}$. Table 1 lists the details concerning the WFPC2 data.

The HST pipeline WFPC2 images were subjected to the usual processing using the $\operatorname{IRAF}^{5}$ and $\mathrm{STSDAS}^{6}$ software packages,

\footnotetext{
5 IRAF is distributed by the National Optical Astronomy Observatory, which is operated by the Association of Universities for Research in Astronomy (AURA), Inc., under cooperative agreement with the National Science Foundation.

6 The Space Telescope Science Data Analysis System (STSDAS) is a product
} of the Space Telescope Science Institute, operated by AURA for NASA. 


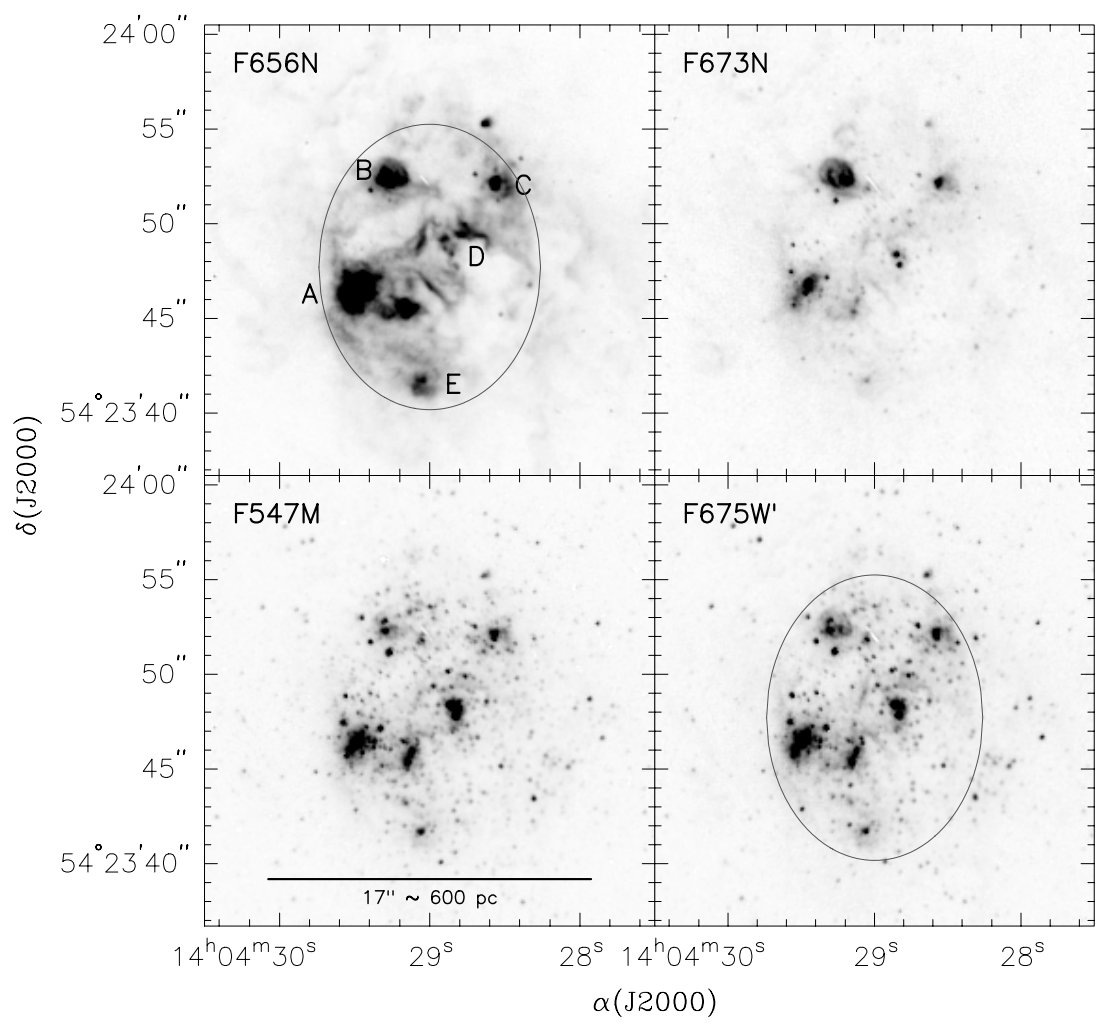

Figure 2. HST WFPC2 images of NGC 5471 in continuum-corrected nebular filters H $\alpha$ (F656N), [S II] (F673N), and emission-line-corrected continuum filters F547M and F675W'. The five knots from Skillman (1985) are marked in the $\mathrm{H} \alpha$ image. An ellipse indicating the core of the region is drawn in the H $\alpha$ and F675W' images.

following the procedures outlined in the HST Data Handbook. We used the multiple exposures to correct for the incidence of cosmic rays as well as to eliminate hot pixels and other defects on the CCD. We finally co-added the images to produce deep mosaics.

Given the luminosity of NGC 5471, its $\mathrm{H} \alpha$ and [S II] emission contributes significantly to the broadband (F675W) image. To eliminate the nebular contribution from the broad band and to produce continuum-free $\mathrm{H} \alpha$ and [S II] emission-line images, we followed the procedure described below. First, we multiplied the images by the PHOTFLAM parameter obtained by synphot to convert from count rates to flux densities, and then divided them by their respective time exposure. From the SYNPHOT task in STSDAS, we determined the filter widths and multiplied the flux densities found by the corresponding filter widths in order to obtain fluxes. The correct filter widths are the filter rectangular width RECTW (Luridiana et al. 2003) defined as the width in Ångstroms of a rectangle with the same total area as the total transmission curve and a height given by the peak in the curve. We then scaled the $\mathrm{H} \alpha$, [S II], and F675W images and performed an iterative subtraction to obtain a new, emission-line free, broadband image $\left(\mathrm{F} 675 \mathrm{~W}^{\prime}\right)$.

Figure 2 shows the $\mathrm{H} \alpha$ image. The five brightest components first noted by Skillman (1985) and designated as A, B, C, D, and $\mathrm{E}$, are distinctly observed in that figure. The presence of a supernova remnant (NGC 5471B) is clearly seen as an intense $\left[\mathrm{S}_{\text {II }}\right]$ emission source in the B-component in the F673N image. Previous works have associated X-ray sources with supernova remnants (SNRs) in M101. Chen et al. (2002) conclude that the energetic SNR in NGC 5471B (first identified by Skillman 1985) was very likely produced by a hypernova, an explosion one or two orders of magnitude brighter than the canonical
Table 2

Journal of TNG/ARNICA Observations of NGC 5471

\begin{tabular}{lccc}
\hline \hline Band & Time (UT) & Duration (s) & Air Mass \\
\hline$J$ & $04: 09: 00$ & $1 \times 60$ & 1.38 \\
$H$ & $01: 03: 08$ & $3 \times 15$ & 1.11 \\
$K_{S}$ & $21: 59: 29$ & $10 \times 5$ & 1.31 \\
\hline
\end{tabular}

$10^{51}$ erg energy explosion associated with a normal SN, although this result has been questioned recently (Jenkins et al. 2004).

\subsection{Near-infrared Photometry}

$J H K_{\text {s }}$ photometry was performed on 2000 April 29, using the $3.58 \mathrm{~m}$ Telescopio Nazionale Galileo (TNG) at the Observatorio del Roque de los Muchachos in La Palma. The camera used was the ARcetri Near Infrared CAmera (ARNICA), with a CCD detector NICMOS $256 \times 256 \mathrm{HgCdTe}$ and gain of $19 e^{-} / \mathrm{ADU}$. The spatial scale was 0.35 pixel $^{-1}$ and the field of view was $90 \times 90 \operatorname{arcsec}^{2}$. The details of these observations are given in Table 2.

The flux calibration of the images was performed using Two Micron All Sky Survey (2MASS) images of NGC 5471, retrieved from the 2MASS Extended Source Catalog. We first measured the integrated flux of the 2MASS images in a circle containing the region and then we used that aperture centered in the same coordinates and covering the same area in the ARNICA images. We obtained the photometric constants comparing the calibrated 2MASS fluxes with those from ARNICA by means of the transformation equations of the two systems.

In order to compare the two sets of optical and NIR data, we aligned the HST and TNG images. We degraded the WFPC2 


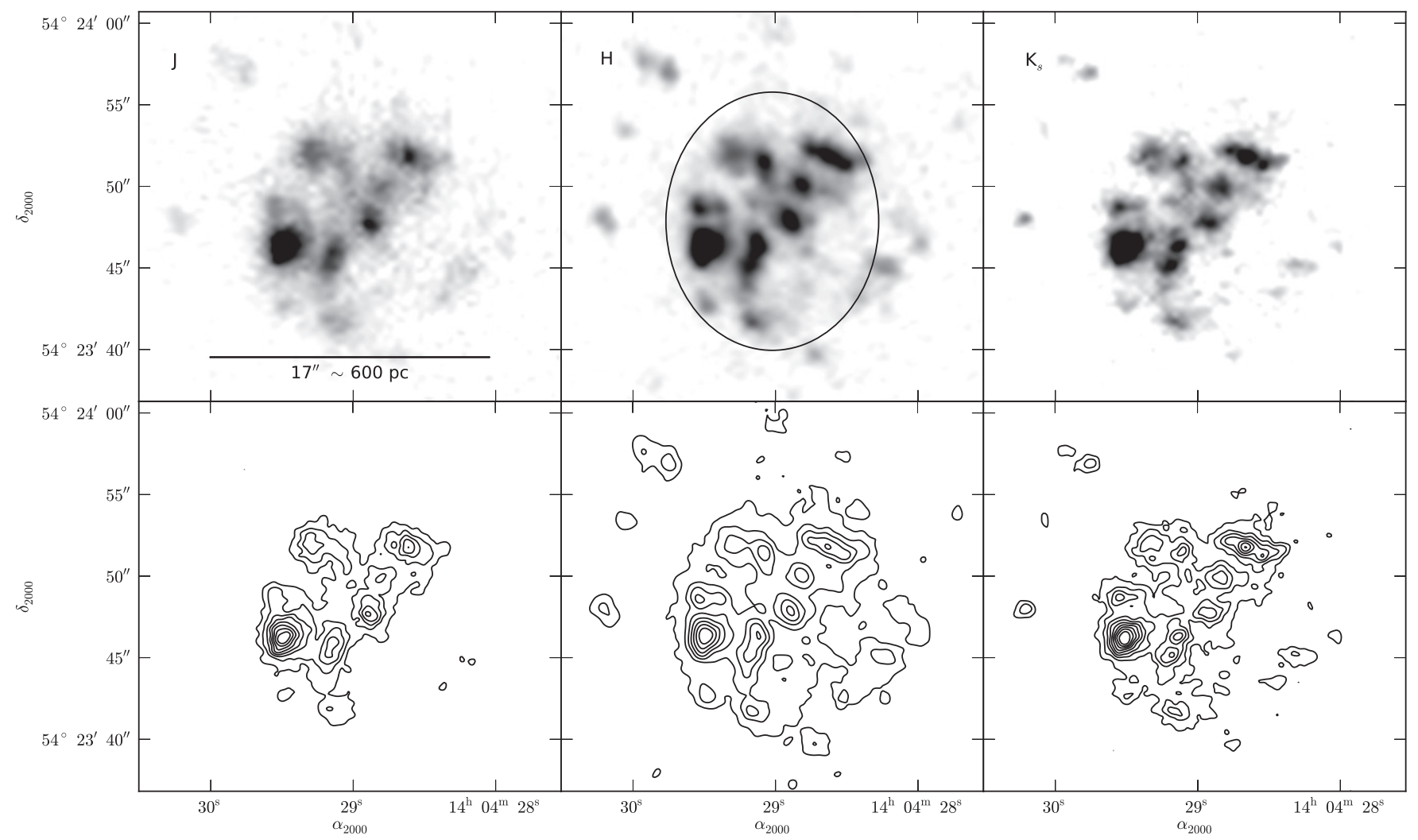

Figure 3. $\mathrm{JHK}_{s}$ gray-scale images and contour plots. The isophotes range from $20 \%$ up to $100 \%$ of the peak value of the image. An ellipse indicating the core of the region is drawn in the $\mathrm{H}$ image.

images to the spatial scale and seeing of the IR ones, then we identified several objects in both data sets and marked them with the IRAF center routine. Next, we aligned the images with the geomap and geotran routines, using the HST images as reference.

Figure 3 shows the images and contour plots of the infrared bands. The multiple knots present in these images are well identified with those in Figure 2 in the F547M and F673W' filters.

\section{RESULTS}

\subsection{Resolved Stellar Photometry}

\subsubsection{WFPC2 Photometry}

The stellar photometric analysis was performed with the HSTphot package (Dolphin 2000a). This package is specifically designed for use with HST WFPC2 images. It uses a library of Tiny Tim (Krist 1995) undersampled PSFs for different locations of the star on the camera and of the star within the pixel, to center the star and to find its magnitude, given in the flight system magnitude. It also contains several programs for general reduction procedures. The first step is to run the mask task to mask out the bad pixels and other image defects identified in the data quality images. The sky computation is made by getsky, which takes all pixels in an annulus around each pixel, determines the sky value, and calculates the sky background map. The next step is to run crmask for cosmic ray removal, which uses the sky file created by getsky. It has the capability of cleaning images that are not perfectly aligned, and it can handle images from different filters. To combine multiple images per filter and to produce a final deep image per filter, the coadd procedure was used. The final step requires the hotpixels procedure, which uses the result from getsky and tries to locate and remove all hot pixels. This is an important step, since hot pixels can create false detections and also, can throw off the PSF solutions.

The hstphot routine was run on the images in the F547M and $\mathrm{F} 675 \mathrm{~W}^{\prime}$ bands. This task performs stellar PSF photometry on multiple images from different filters, including alignment and aperture corrections as well as PSF modifications to correct for errors of geometric distortion via the Holtzman et al. (1995) distortion correction equations and the 34th row error, noted by Shaklan et al. (1995; see also Anderson \& King 1999), and correction for charge transfer inefficiency (Dolphin $2000 \mathrm{~b}$ ). There are several parameters to use during the run of hstphot. We enabled the determination of the "local sky" (from now on, nebular case), because of the rapidly varying background of NGC 5471, but we also used, in a different run, the usual (stellar) fit done by getsky. We made a crosscorrelation between the nebular and stellar lists of stars in order to match them and find the sources detected using both types of sky determination. To have a better estimate of the sky for those matched stars, we took as magnitude the mean of both measurements $m=\left(m_{\text {nebular }}+m_{\text {stellar }}\right) / 2$ and as error

$$
\sigma_{m}=\max \left(\sigma_{m_{\mathrm{n}}}, \sigma_{m_{\mathrm{s}}}, \frac{\left|m_{\mathrm{n}}-\mathrm{ms}\right|}{2}\right) .
$$

The final list of stars is made up of (1) matched stars, (2) objects from the stellar list not included in the matched star list (best detected in less nebulous regions), and (3) stars from the nebular list with no companion in the stellar list.

We selected "good stars" from the hstphot output. Object types were classified as "good star," "possible unresolved binary," "bad star," "single-pixel cosmic ray or hot pixel," and 
"extended object." Less than $10 \%$ of the stars were not classified as "good stars." To ensure that we only have point-like sources, we also used the "sharpness" parameter and a measure of the quality of the fit $\left(\chi^{2}\right)$ to reject false star detections in regions with structured nebulosity or artifacts. The final number of stars detected in the WF3 chip with these parameters in both filters was 1261 .

\subsubsection{Selection Effects}

Completeness. In order to face the study of the star formation history (SFH) of an object and simulate synthetic diagrams, it is compulsory to undertake a completeness analysis. A thorough description of the completeness analysis can be found in Stetson \& Harris (1988) and Aparicio \& Gallart (1995). Below we briefly describe its basis and main steps.

Incompleteness is mainly due to two factors: overlapping of stellar profiles in crowded regions and background noise. These effects are more important for faint stars in dense stellar regions. Consequently, in a crowded cluster, incompleteness will depend on apparent magnitude and spatial position. The best way to account for crowding effects is to add artificial stars to the images and to subject them to the same sample selection as for real stars. The number of injected stars must be sufficiently high as to provide acceptable statistical accuracy, but not so high as to change the level of crowding. To avoid overcrowding effects, no more than some $10 \%$ of the number of real stars should be added as artificial stars. These new artificial images (synthetic frames) are processed in the same way as the real ones, and the magnitudes of all the stars are obtained following the same criteria and using the same parameters. At this point, we will have three different tables: (1) the first one containing magnitudes, positions, and colors of the real stars (real stars); (2) the second one including all the data for the injected stars, which are known beforehand; and (3) the third list containing the data from the synthetic frames.

The synthetic table is then cross-correlated with the other two (real + injected), producing a new table (completeness) containing the stars matched from the artificial list. Therefore, in this final list we have the initial and recovered magnitudes and colors of the injected stars as well as their coordinates. The table will also contain stars which have not been paired, and a flag indicating that they have been lost. The ratio between the number of simulated stars recovered and injected provides the estimate of the completeness factor for each magnitude bin.

A grid of artificial stars was generated on a two-dimensional (2D) color-magnitude diagram (CMD) and distributed according to the flux of the images with an artificial star routine provided by HSTphot. The parameters of the routine are the minimum and maximum of the measured color and magnitude. The magnitude steps used were multiple of 0.5 , while color steps are by 0.25 . We asked the routine to distribute artificial stars on the CMD in accordance with the number observed. Approximately 40,000 fake stars were added in each image (at different trials, in order to leave the crowding conditions unaltered) and were given random magnitudes and colors in the observed range. We estimated the completeness in two different regimes of crowding: inside the core of the region (inner ellipse in Figure 4) and in the rest of the image. For the core, the $50 \%$ completeness of the F547M filter luminosity function derived on the basis of the CMD data is reached at $24.8 \mathrm{mag}$, while for the $\mathrm{F} 675 \mathrm{~W}^{\prime}$ filter it is $24.3 \mathrm{mag}$. For the rest of the image, the $50 \%$ completeness of the F547M filter is reached at $25.2 \mathrm{mag}$, while for the $\mathrm{F}_{675 \mathrm{~W}^{\prime}}$ filter it is $24.7 \mathrm{mag}$.

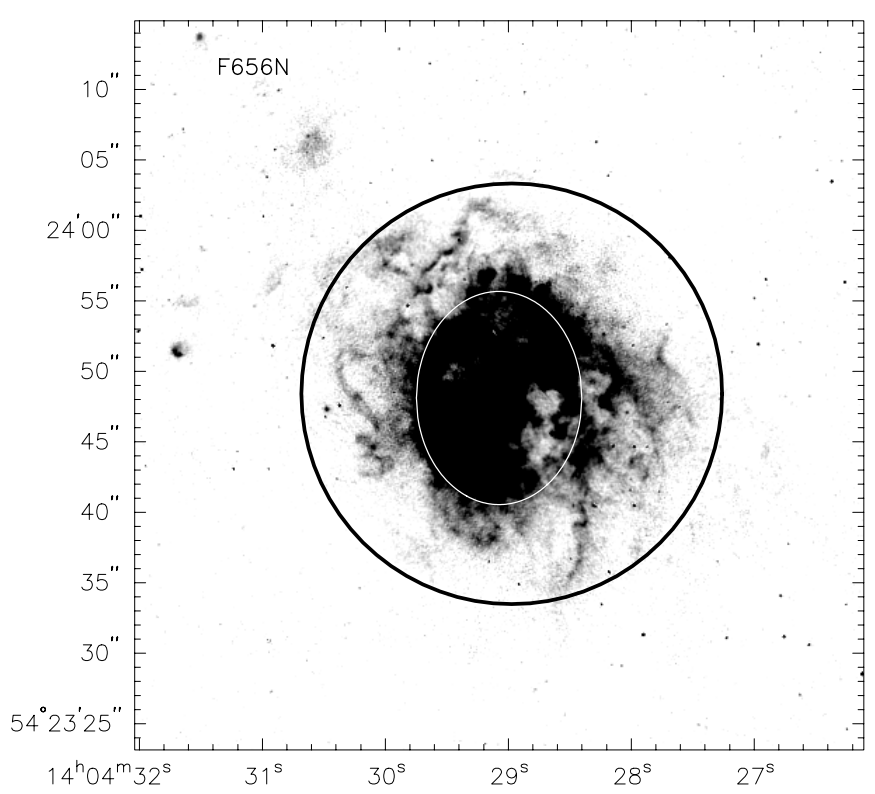

Figure 4. Continuum-free $\mathrm{H} \alpha$ image of NGC 5471. The circular aperture of $15^{\prime \prime}$ where the integrated photometry was performed to obtain the total flux is overplotted together with the inner ellipse defining the core of the region.

Field star subtraction. Statistical decontamination has been widely used to accomplish the field star subtraction of CMDs. The idea is very simple: our region of study, NGC 5471, consists of the sum of the intrinsic population, IP, and the contaminating population, FS, and therefore our CMD shows both populations. The field star contribution can be estimated using the CMD of an adjacent field $\mathrm{FS}^{\prime}$ containing only foreground and background stars, assuming that it is statistically representative of FS. Accordingly, the FS contribution may be removed by comparing the local density of stars in the two diagrams (Bellazzini et al. 1999):

$$
\mathrm{CMD}(\mathrm{IP}+\mathrm{FS})-\mathrm{CMD}\left(\mathrm{FS}^{\prime}\right) \sim \mathrm{CMD}(\mathrm{IP})
$$

As NGC 5471 is centered in the WF3, we assume that all of the stars in the other chips are field stars. This is not completely true because this GEHR is in the outskirts of an M101 arm, where a few traces of star formation activity can still be found. Nevertheless, they are easily detected and no more than 20 stars in the WF2 (the only chip used to determine the FS population) were found over the chip. The rest of the stars showed a homogeneous distribution and were considered a sample of field stars. A CMD(IP+FS) was constructed for a region inside a circular aperture of $15^{\prime \prime}$ which includes the core+halo structure of the region.

To perform the statistical decontamination, we followed the procedure described by Mighell et al. (1996) and Bellazzini et al. (1999). For a given star in the CMD(IP+FS) with coordinates $\left[m \pm \sigma_{m} ; c \pm \sigma_{c}\right]$, we count how many stars $\left(N_{\mathrm{IP}+\mathrm{FS}}\right)$ can be found within the ellipse of axes $\left[\max \left(\sigma_{m}, 0.1\right) ; \max \left(\sigma_{c}, 0.1\right)\right]$ in the original diagram. We also counted the number of stars $\left(N_{\mathrm{FS}^{\prime}}\right)$ found in the field $\mathrm{CMD}\left(\mathrm{FS}^{\prime}\right)$ within the same ellipse centered at the same coordinates.

The probability $P$ that a given star in the $\mathrm{CMD}(\mathrm{IP}+\mathrm{FS})$ is actually a member of NGC 5471 is defined as follows:

$$
P=1-\min \left(\frac{\alpha N_{\mathrm{FS}^{\prime}}}{N_{\mathrm{IP}+\mathrm{FS}}}, 1.0\right),
$$

where $\alpha$ is the ratio of the area of the IP+FS field (a circular 


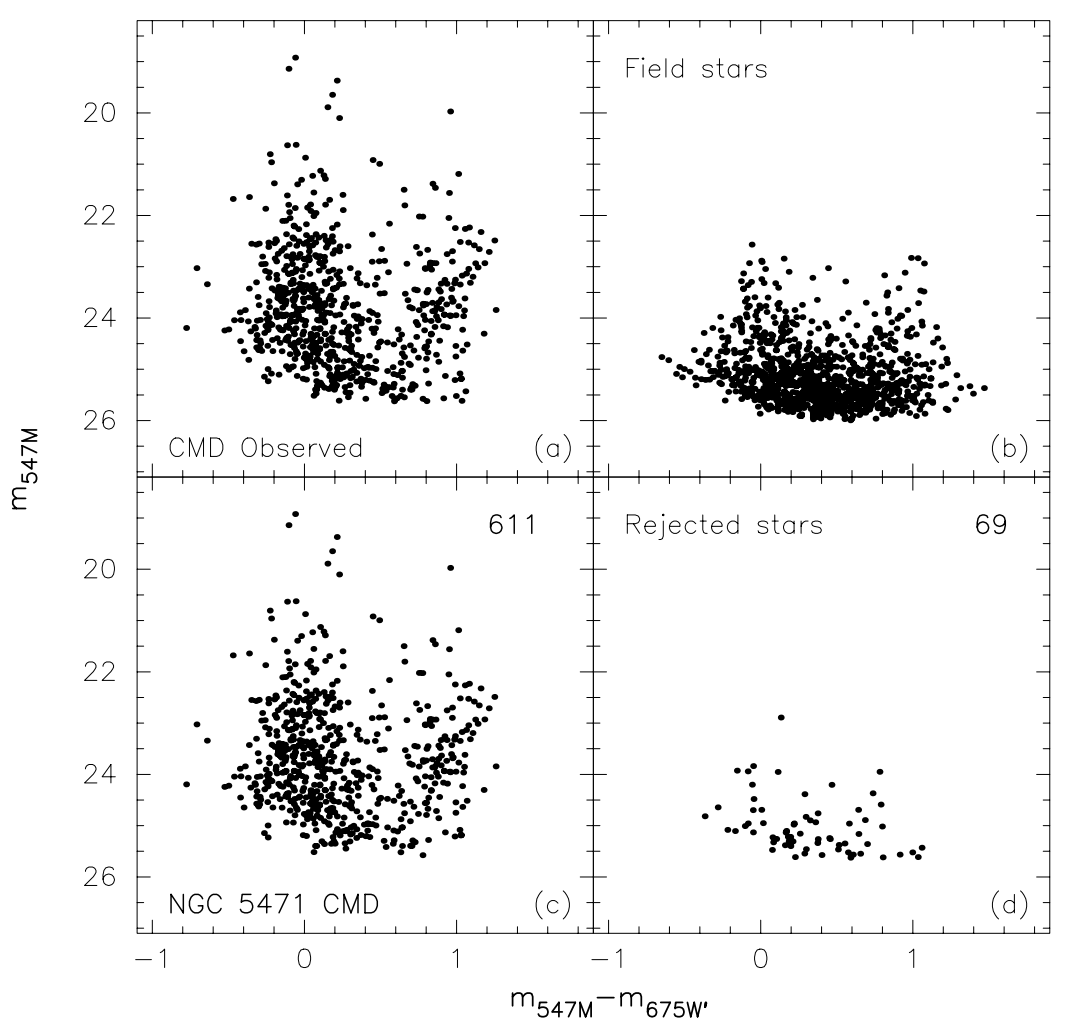

Figure 5. Decontamination of the NGC 5471 observed CMD (panel (a)) using the field stars CMD (panel (b)). The decontaminated NGC 5471 CMD is shown in panel (c) (610 stars) while the CMD of the rejected stars is displayed in the panel (d) (73 stars).

aperture of $15^{\prime \prime}$ ) to the area of the $\mathrm{FS}^{\prime}$ field (in this case, the whole WF2 chip). We can determine the probable membership of this star by comparing $P$ with an uniform random number $0<P^{\prime}<1$; if $P^{\prime} \leqslant P$ then the star is accepted as a member of NGC 5471; otherwise it is rejected and considered to be a field star. Since this cleaning method is probabilistic in nature, a decontaminating trial represents only one out of an infinite number of different realizations of the cleaned CMD for NGC 5471.

Figure 5 shows the result of this process of decontamination for the CMD of NGC 5471. Panel (a) displays the CMD observed (with both intrinsic and field stars), panel (b) shows the field stars, panel (c) displays the decontaminated CMD of NGC 5471 and panel (d) shows the CMD of the rejected stars. Table 3 shows the magnitudes of the first 20 stars of the resulting clean CMD of panel (c); the full table can be accessed in the online version of the journal.

\subsubsection{Isochrones}

To study the CMD, we use the isochrone set that corresponds to the grid by Girardi et al. (2002), ${ }^{7}$ covering the age range $6.60<\log t<10.25$ and mass range $0.15-100 M_{\odot}$, based on the $Z=0.004\left(1 / 5 Z_{\odot}\right)$ models (see the end of Section 3.2 for a discussion on the choice of the metallicity) from Girardi et al. (2000) and Bertelli et al. (1994) that include overshooting and a simple synthetic evolution of the thermally pulsing asymptotic giant branch (Girardi \& Bertelli 1998). Atmosphere models are from the BaSeL 3.1 WLBC 99 corrected library (Westera et al. 2002) at the same metallicity as the isochrone set.

The procedure is as follows. (1) We obtained the magnitudes in the HST filters for all stellar models in the atmosphere grid.

\footnotetext{
7 Available at http://pleiadi.pd.astro.it/.
}

(2) For each point in the isochrone we obtained the stellar flux in each of the bands by a linear interpolation in the $\log g-\log T_{\text {eff }}$ grid. (3) The final luminosity was obtained by multiplying by the square radius of the star. For $\log g-\log T_{\text {eff }}$ values not covered by the atmosphere grid, the boundary values have been used with a caution flag. In terms of the present work, these stars do not modify the results obtained.

In Figure 6, we show the final CMD (corrected for field star contamination). The $50 \%$ completeness limit is also plotted, and five isochrones from 4 to $70 \mathrm{Myr}$ are shown in color. The righthand side scale gives the absolute magnitude corresponding to a distance of 7.2 Mpc.

\subsection{Cluster Photometry}

We have derived the integrated magnitudes and colors in 11 different apertures covering the main components in the core of NGC 5471. The apertures were chosen and defined in the $H$-band image, maximizing the area in order to include the features of all images, since there were some small offsets in the position and intensity of the knots in the optical and infrared images, respectively. Then, the same polygons were used for the $J, K_{s}$, and HST images. Polygons were marked by using the IRAF task polymark, and the integrated magnitudes were computed using the IRAF task polyphot. The integrated magnitudes of the HST images are given in the VEGAMAG system. The polygons defined are shown in Figure 7.

The integrated magnitudes and colors derived in each area were corrected for the Galactic foreground extinction toward M101 using the color excess $E(B-V)=0.01$ (Schlegel et al. 1998), although its effect is negligible. The absorption coefficients for the five bands were derived assuming $R_{V}=$ $A_{V} / E(B-V)=3.1$ and using the $A_{\lambda}$ curve from Cardelli et al. (1989). The uncertainty of the individual fluxes have 


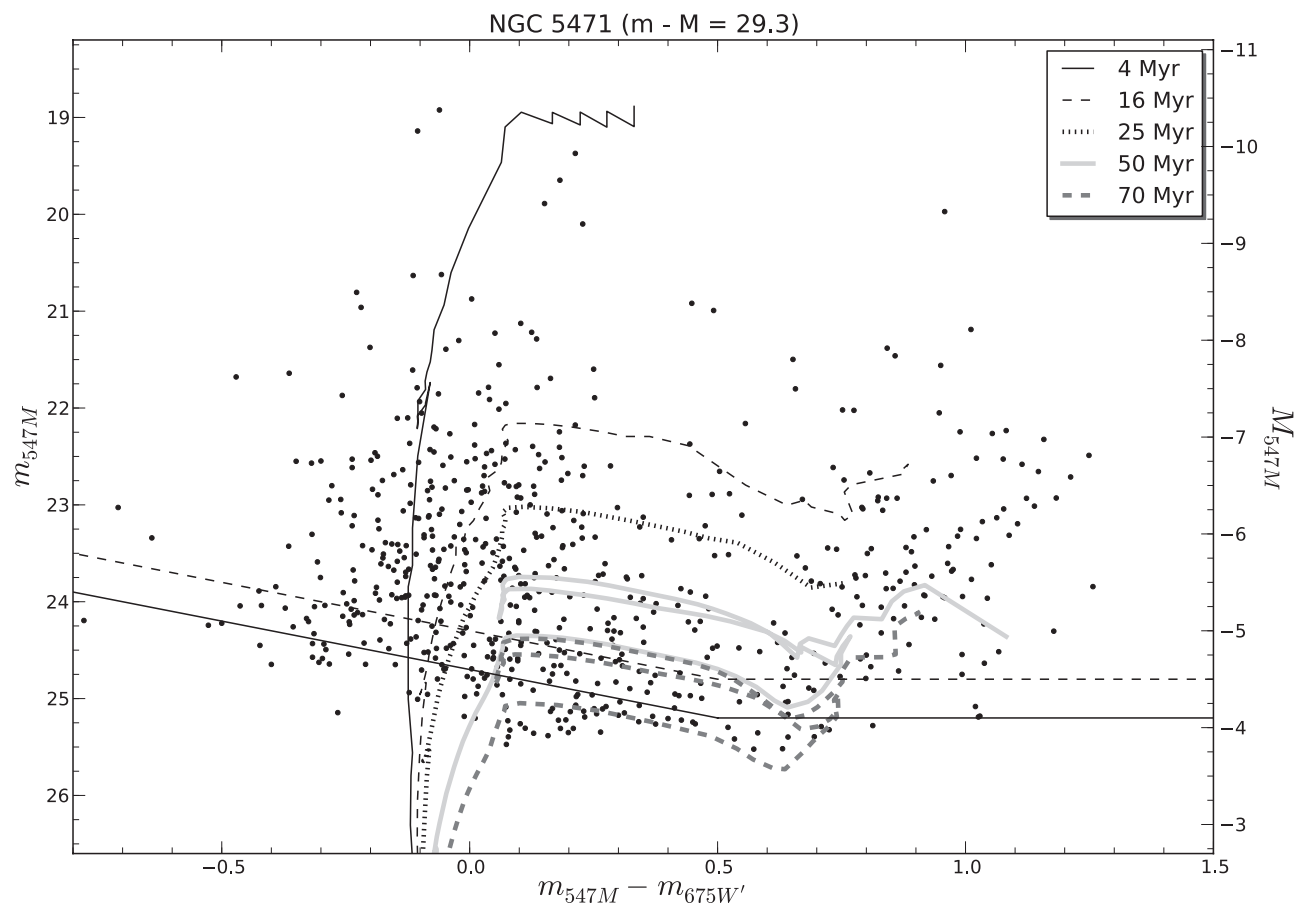

Figure 6. Final CMD corrected for field star contamination, together with five isochrones from 4 to 70 Myr. The line across the bottom shows the completeness limit at the 50\% level as derived from false star tests for the core (dashed line) and for the rest of the image (solid line). The right-hand side scale gives the absolute magnitudes corresponding to a distance of $7.2 \mathrm{Mpc}$.

(A color version of this figure is available in the online journal.)

Table 3

Stellar Photometry

\begin{tabular}{|c|c|c|c|c|c|c|c|}
\hline ID & $\begin{array}{c}X \\
\text { (pixel) } \\
\end{array}$ & $\begin{array}{c}Y \\
\text { (pixel) } \\
\end{array}$ & $\begin{array}{l}\text { R.A. } \\
\text { (J2000) }\end{array}$ & $\begin{array}{c}\text { Decl. } \\
(\mathrm{J} 2000)\end{array}$ & $\begin{array}{c}m_{\mathrm{F} 547 \mathrm{M}} \\
(\mathrm{mag})\end{array}$ & $\begin{array}{c}m_{\mathrm{F} 675 \mathrm{~W}^{\prime}} \\
(\mathrm{mag})\end{array}$ & $\begin{array}{c}m_{\mathrm{F} 547 \mathrm{M}}-m_{\mathrm{F} 675 \mathrm{~W}^{\prime}} \\
(\mathrm{mag})\end{array}$ \\
\hline 1 & 359.56 & 562.57 & 14:04:27.50 & $54: 23: 41.53$ & $24.24 \pm 0.07$ & $23.45 \pm 0.05$ & $0.79 \pm 0.09$ \\
\hline 2 & 324.75 & 512.37 & $14: 04: 27.56$ & $54: 23: 47.59$ & $24.57 \pm 0.10$ & $23.93 \pm 0.07$ & $0.65 \pm 0.12$ \\
\hline 3 & 352.44 & 544.56 & $14: 04: 27.57$ & $54: 23: 43.36$ & $24.05 \pm 0.07$ & $23.95 \pm 0.06$ & $0.09 \pm 0.09$ \\
\hline 4 & 332.62 & 514.12 & $14: 04: 27.61$ & $54: 23: 46.96$ & $24.59 \pm 0.09$ & $24.88 \pm 0.19$ & $-0.30 \pm 0.21$ \\
\hline 5 & 378.56 & 569.56 & $14: 04: 27.62$ & $54: 23: 39.79$ & $24.04 \pm 0.06$ & $23.62 \pm 0.06$ & $0.42 \pm 0.08$ \\
\hline 6 & 323.33 & 496.44 & $14: 04: 27.66$ & $54: 23: 48.91$ & $24.07 \pm 0.06$ & $24.13 \pm 0.12$ & $-0.06 \pm 0.14$ \\
\hline 7 & 373.56 & 553.15 & $14: 04: 27.69$ & $54: 23: 41.37$ & $24.18 \pm 0.07$ & $23.24 \pm 0.05$ & $0.94 \pm 0.09$ \\
\hline 8 & 293.29 & 455.69 & 14:04:27.69 & $54: 23: 53.94$ & $23.62 \pm 0.05$ & $22.57 \pm 0.03$ & $1.05 \pm 0.06$ \\
\hline 9 & 351.67 & 523.45 & $14: 04: 27.71$ & $54: 23: 45.04$ & $23.85 \pm 0.07$ & $23.87 \pm 0.11$ & $-0.02 \pm 0.13$ \\
\hline 10 & 290.75 & 449.59 & $14: 04: 27.71$ & $54: 23: 54.57$ & $23.86 \pm 0.06$ & $23.02 \pm 0.04$ & $0.84 \pm 0.07$ \\
\hline 11 & 368.80 & 540.45 & $14: 04: 27.74$ & $54: 23: 42.65$ & $23.14 \pm 0.03$ & $23.24 \pm 0.04$ & $-0.10 \pm 0.05$ \\
\hline 12 & 346.68 & 513.49 & $14: 04: 27.74$ & $54: 23: 46.12$ & $25.26 \pm 0.15$ & $24.89 \pm 0.17$ & $0.38 \pm 0.23$ \\
\hline 13 & 381.63 & 555.86 & $14: 04: 27.75$ & $54: 23: 40.66$ & $24.08 \pm 0.07$ & $24.18 \pm 0.11$ & $-0.10 \pm 0.13$ \\
\hline 14 & 360.90 & 530.61 & $14: 04: 27.75$ & $54: 23: 43.91$ & $23.96 \pm 0.06$ & $24.09 \pm 0.09$ & $-0.14 \pm 0.10$ \\
\hline 15 & 318.82 & 469.76 & $14: 04: 27.81$ & $54: 23: 51.25$ & $25.22 \pm 0.15$ & $25.04 \pm 0.20$ & $0.18 \pm 0.26$ \\
\hline 16 & 312.47 & 461.48 & $14: 04: 27.81$ & $54: 23: 52.29$ & $23.20 \pm 0.04$ & $23.22 \pm 0.04$ & $-0.02 \pm 0.05$ \\
\hline 17 & 291.51 & 435.95 & $14: 04: 27.81$ & $54: 23: 55.57$ & $25.38 \pm 0.21$ & $25.23 \pm 0.19$ & $0.16 \pm 0.28$ \\
\hline 18 & 359.87 & 517.56 & $14: 04: 27.83$ & $54: 23: 44.98$ & $24.20 \pm 0.09$ & $24.26 \pm 0.10$ & $-0.06 \pm 0.13$ \\
\hline 19 & 354.84 & 509.85 & $14: 04: 27.84$ & $54: 23: 45.89$ & $24.02 \pm 0.06$ & $23.67 \pm 0.05$ & $0.34 \pm 0.08$ \\
\hline 20 & 319.56 & 460.53 & $14: 04: 27.88$ & $54: 23: 51.91$ & $24.45 \pm 0.08$ & $24.87 \pm 0.20$ & $-0.42 \pm 0.22$ \\
\hline
\end{tabular}

Notes. Stellar photometry.

(This table is available in its entirety in machine-readable and Virtual Observatory (VO) forms in the online journal. A portion is shown here for guidance regarding its form and content.)

been computed including the Poisson photon count error, the uncertainty on the background setting, and the photometric calibration uncertainty. The results of the integrated photometry are shown in Table 4.

We have also measured the $\mathrm{H} \alpha$ flux for each polygon. To measure the integrated $\mathrm{H} \alpha$ flux of the whole region, we have decontaminated the F656N image from the broadband F675W contribution. We have then plotted the contour at $3 \sigma$ from the sky (with isophotal flux $6.3 \times 10^{-15} \mathrm{erg} \mathrm{cm}^{-2} \mathrm{~s}^{-1} \operatorname{arcsec}^{-1}$ ) and found the circular aperture centered in the region that best fits that isophotal contour. The radius of this aperture is 15 arcsec from the center (see Figure 4). The flux measured inside this circular aperture is $(3.65 \pm 0.17) \times 10^{-12} \mathrm{erg} \mathrm{cm}^{-2} \mathrm{~s}^{-1}$. We have used the PHOTFLAM value given by synphot and 


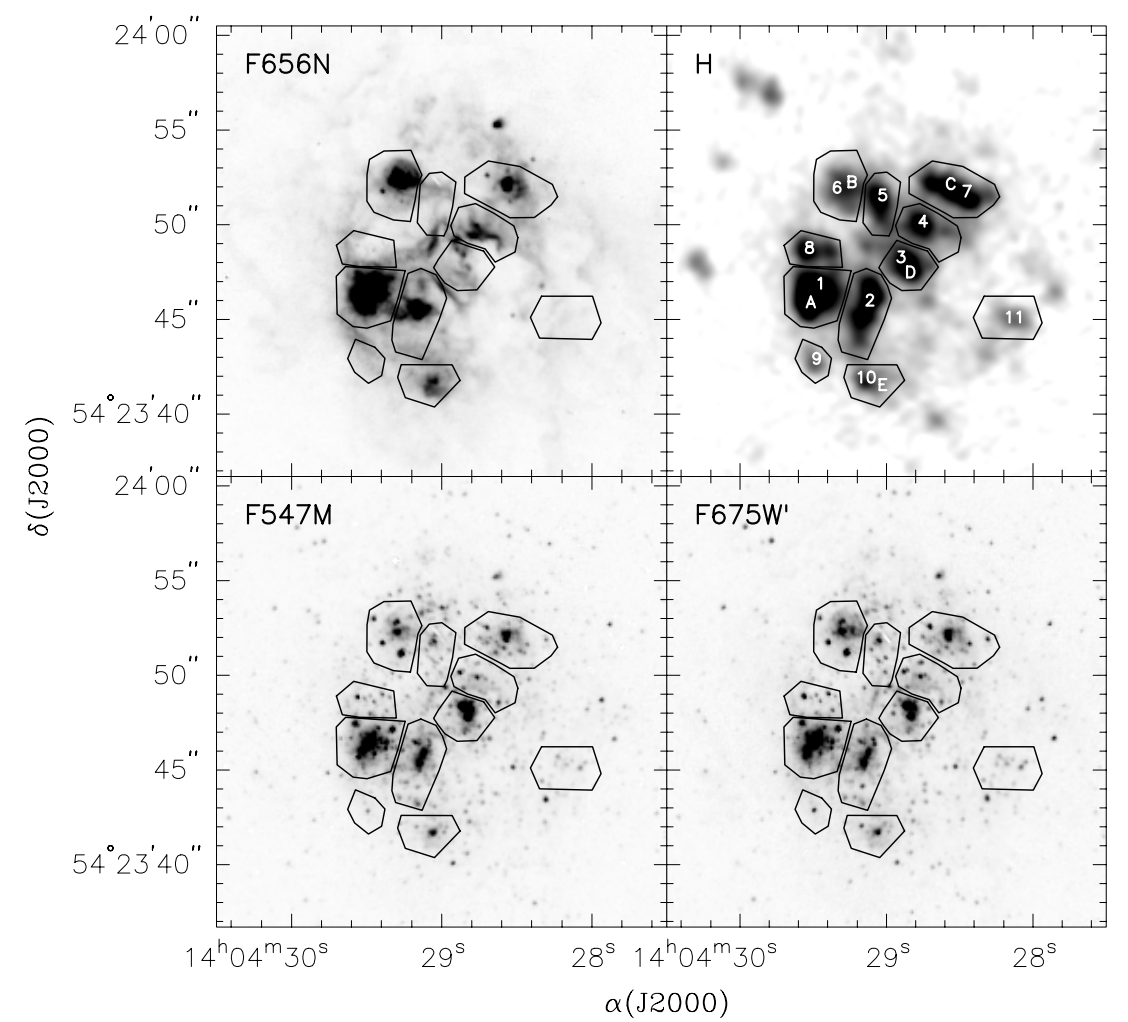

Figure 7. Plot of the 11 polygons, limiting the different areas where the integrated magnitudes are computed. The numbers belong to our own definition while the A, B, C, D, and E letters are the knots identified by Skillman (1985). The hypernova is located in knot 6 .

Table 4

Integrated Photometry

\begin{tabular}{|c|c|c|c|c|c|c|}
\hline Aperture & $J$ & $H$ & $K_{s}$ & $m_{547 \mathrm{M}}$ & $m_{675 \mathrm{~W}}$ & $m_{675 \mathrm{~W}^{\prime}}$ \\
\hline N5471-1 & $16.43 \pm 0.11$ & $16.26 \pm 0.12$ & $16.03 \pm 0.14$ & $16.66 \pm 0.06$ & $15.56 \pm 0.06$ & $16.30 \pm 0.06$ \\
\hline N5471-2 & $16.90 \pm 0.11$ & $16.57 \pm 0.12$ & $16.48 \pm 0.14$ & $17.21 \pm 0.06$ & $16.30 \pm 0.06$ & $16.94 \pm 0.06$ \\
\hline N5471-3 & $17.35 \pm 0.11$ & $17.09 \pm 0.12$ & $17.12 \pm 0.14$ & $17.22 \pm 0.06$ & $16.84 \pm 0.06$ & $17.11 \pm 0.06$ \\
\hline N5471-4 & $17.52 \pm 0.11$ & $17.08 \pm 0.12$ & $16.99 \pm 0.14$ & $18.17 \pm 0.06$ & $17.07 \pm 0.06$ & $17.77 \pm 0.06$ \\
\hline N5471-5 & $17.83 \pm 0.11$ & $17.28 \pm 0.12$ & $17.27 \pm 0.14$ & $18.58 \pm 0.06$ & $17.59 \pm 0.06$ & $18.16 \pm 0.06$ \\
\hline N5471-6 & $17.34 \pm 0.11$ & $17.21 \pm 0.12$ & $17.00 \pm 0.14$ & $17.62 \pm 0.06$ & $16.41 \pm 0.06$ & $17.12 \pm 0.06$ \\
\hline N5471-7 & $16.98 \pm 0.11$ & $16.59 \pm 0.12$ & $16.47 \pm 0.14$ & $17.56 \pm 0.06$ & $16.49 \pm 0.06$ & $17.10 \pm 0.06$ \\
\hline N5471-8 & $17.89 \pm 0.11$ & $17.41 \pm 0.12$ & $17.39 \pm 0.14$ & $18.70 \pm 0.06$ & $17.68 \pm 0.06$ & $18.22 \pm 0.06$ \\
\hline N5471-9 & $19.15 \pm 0.13$ & $18.63 \pm 0.13$ & $18.63 \pm 0.15$ & $20.23 \pm 0.06$ & $18.71 \pm 0.06$ & $19.54 \pm 0.06$ \\
\hline N5471-10 & $18.14 \pm 0.11$ & $17.80 \pm 0.12$ & $17.57 \pm 0.15$ & $18.63 \pm 0.06$ & $17.45 \pm 0.06$ & $18.22 \pm 0.06$ \\
\hline N5471-11 & $18.55 \pm 0.12$ & $18.00 \pm 0.12$ & $18.11 \pm 0.15$ & $19.27 \pm 0.06$ & $18.40 \pm 0.06$ & $18.84 \pm 0.06$ \\
\hline NGC 5471 & $14.14 \pm 0.10$ & $13.84 \pm 0.12$ & $13.74 \pm 0.14$ & $14.67 \pm 0.06$ & $13.62 \pm 0.06$ & $14.27 \pm 0.06$ \\
\hline
\end{tabular}

Notes. Integrated magnitudes and $\mathrm{H} \alpha$ flux for the 11 regions of NGC 5471. Magnitudes from $H S T$ are given in the VEGAMAG system. Results for $m_{675 \mathrm{~W}^{\prime}}$ column were measured from the emission-line free F675W image. The last row belongs to the integrated magnitude of the whole region.

a value of RECTW of $28.3 \AA$. Measured $\mathrm{H} \alpha$ fluxes for each of the polygonal selections and the parameters derived from them are given in Table 5 . The effective radius $\left(R_{\text {eff }}\right)$ of a polygon is defined as the radius of a circular aperture of the same area.

Since GALEX images do not have the necessary resolution, we were not able to get the fluxes for each polygon. As in the case of the nebular flux, we measured the FUV and NUV fluxes inside $15^{\prime \prime}$ radii apertures centered in NGC 5471. We obtain an FUV flux of $(9.9 \pm 0.1) \times 10^{-14} \mathrm{erg} \mathrm{cm}^{-2} \mathrm{~s}^{-1} \AA^{-1}(14.19 \pm 0.05$ $\mathrm{AB}$ magnitudes) and an NUV flux of $(4.5 \pm 0.1) \times 10^{-14} \mathrm{erg}$ $\mathrm{cm}^{-2} \mathrm{~s}^{-1} \AA^{-1}$ (14.24 $\pm 0.05 \mathrm{AB}$ magnitudes).

In order to use these values in the analysis, we need to transform AB magnitudes to the VEGAMAG system. The transformation formula is (Maíz Apellániz 2007)

$$
\mathrm{AB}_{p}=\left(m_{p}-\mathrm{ZP}_{p}\right)+\mathrm{AB}_{p}(\text { Vega }),
$$

where $\mathrm{AB}_{p}$ is the magnitude of the object in the $\mathrm{AB}$ system and filter passband $p, m_{p}$ is the magnitude of the object in filter $p$ using Vega as reference spectral energy distribution (SED), $\mathrm{ZP}_{p}$ is the zero point of the $p$ filter in the Vega system, and $\mathrm{AB}_{p}(\mathrm{Vega})$ is the $\mathrm{AB}$ magnitude of Vega in filter $p$. This magnitude is given by

$$
\mathrm{AB}_{p}(\text { Vega })=-2.5 \log _{10}\left(\frac{\int P_{p}(\lambda) f_{\lambda, \mathrm{Vega}}(\lambda) \lambda d \lambda}{\int P_{p}(\lambda)\left(c f_{v, \mathrm{AB}} / \lambda\right) d \lambda}\right),
$$

where $P_{p}(\lambda)$ is the dimensionless filter sensitivity function of 
Table 5

Nebular Photometry

\begin{tabular}{|c|c|c|c|c|c|}
\hline Aperture & $\begin{array}{l}R_{\text {eff }} \\
(\mathrm{pc})\end{array}$ & $\begin{array}{c}F(\mathrm{H} \alpha) \\
\left(10^{-13} \mathrm{erg} \mathrm{s}^{-1} \mathrm{~cm}^{-2}\right)\end{array}$ & $\begin{array}{c}L(\mathrm{H} \alpha) \\
\left(10^{39} \mathrm{erg} \mathrm{s}^{-1}\right)\end{array}$ & $\begin{array}{c}Q\left(\mathrm{H}^{0}\right) \\
\left(10^{51} \mathrm{~s}^{-1}\right)\end{array}$ & $\begin{array}{c}M \mathrm{H}_{\mathrm{II}} \\
\left(10^{4} M_{\odot}\right)\end{array}$ \\
\hline NGC 5471-1 & 61.1 & $6.63 \pm 0.31$ & 4.1 & 4.1 & 13.0 \\
\hline NGC 5471-2 & 60.9 & $3.07 \pm 0.14$ & 1.9 & 1.9 & 6.1 \\
\hline NGC 5471-3 & 47.0 & $0.93 \pm 0.04$ & 0.6 & 0.6 & 1.9 \\
\hline NGC 5471-4 & 51.2 & $1.59 \pm 0.07$ & 1.0 & 1.0 & 3.2 \\
\hline NGC 5471-5 & 46.0 & $0.79 \pm 0.04$ & 0.5 & 0.5 & 1.6 \\
\hline NGC 5471-6 & 58.9 & $2.42 \pm 0.11$ & 1.5 & 1.5 & 4.8 \\
\hline NGC 5471-7 & 63.1 & $2.34 \pm 0.11$ & 1.5 & 1.4 & 4.7 \\
\hline NGC 5471-8 & 43.5 & $0.72 \pm 0.03$ & 0.5 & 0.4 & 1.4 \\
\hline NGC 5471-9 & 34.0 & $0.38 \pm 0.02$ & 0.2 & 0.2 & 0.8 \\
\hline NGC 5471-10 & 46.2 & $1.22 \pm 0.06$ & 0.8 & 0.8 & 2.4 \\
\hline NGC 5471-11 & 53.6 & $0.31 \pm 0.01$ & 0.2 & 0.2 & 0.6 \\
\hline NGC 5471 & 525 & $36.5 \pm 1.70$ & 23 & 22 & 73 \\
\hline
\end{tabular}

Notes. Integrated $\mathrm{H} \alpha$ flux and derived parameters for the 11 regions of NGC 5471. $R_{\text {eff }}$ is the effective radius of the polygon. The last row belongs to the integrated values of the whole region.

filter $p, f_{\lambda, \text { Vega }}(\lambda)$ is the Vega spectrum, and $f_{\nu, \mathrm{AB}}=3.63079 \times$ $10^{-20} \mathrm{erg} \mathrm{s}^{-1} \mathrm{~cm}^{-2} \mathrm{~Hz}^{-1}$ is the reference SED of the AB system.

We have used FUV and NUV filter passbands to calculate the $\mathrm{AB}$ magnitudes of Vega by means of Equation (2). As the zero points for $G A L E X$ are $\mathrm{ZP}=0.000$ in both bands, we have

$$
m_{\mathrm{FUV}}=\mathrm{AB}_{\mathrm{FUV}}-2.073
$$

$$
m_{\mathrm{NUV}}=\mathrm{AB}_{\mathrm{NUV}}-1.647 .
$$

Finally, the GALEX VEGAMAG magnitudes of NGC 5471 used below are $m_{\mathrm{FUV}}=12.12$ and $m_{\mathrm{NUV}}=12.59$.

Although a complete analysis of the stellar populations is better characterized by the CMD study, integrated photometry provides additional information from wavelengths where CMD studies are not feasible. However, the usual methodology to infer properties from clusters based on their integrated light is not trivial for low-mass clusters and we need to be sure that the procedure is valid before the use of any analysis tool. The first trivial test is that cluster luminosity fulfills the bolometric assumptions implicit in the synthetic photometry (cluster luminosities larger than the lowest-luminosity limit (LLL); Cerviño \& Luridiana 2004). This basically establishes when the cluster luminosity in a given wavelength/filter may be produced by just an individual star. ${ }^{8}$ The mean luminosity of a cluster obtained by population synthesis models can be safely used when the cluster is brighter than 10 times the LLL or 2.5 in magnitude units (Cerviño \& Luridiana 2004).

The results of the LLL analysis are shown in Figure 8, where the ages are just first-order estimations when LLL and additional limitations in the analysis are not taken into account. The figure shows that, with the exception of a few clusters, most of the clusters do not fulfill the $10 \times$ LLL safety limit. Thus, formally, the analysis based on the mean values obtained from synthesis models should be considered to be preliminary and used with a cautionary note, as some could be biased by contributions from individual stars.

With this limitation in mind and taking into account that the results could be reinterpreted, we have analyzed the integrated

\footnotetext{
8 The LLL just establishes this possibility, but it does not provide more
} information about whether it is really the case. photometric measurements with the help of CHORIZOS, ${ }^{9}$ a code written in IDL by Maíz Apellániz (2004). This code compares photometric data with model SEDs, calculating the likelihood for the full parameter range specified, allowing for the identification of multiple solutions. CHORIZOS can handle SED families for stellar atmospheres and the mean SED obtained by synthesis models ${ }^{10}$ (Starburst99; Leitherer et al. 1999) and uses $\chi^{2}$ minimization to find all models compatible with the observed data in a ( 2 intrinsic +2 extrinsic)-dimensional parameter space. It includes a wide list of filters for different systems and allows for published magnitudes to be used directly. For a set of objects with $M+1$ measured magnitudes (with independent uncertainties), derived $M$-independent colors, and a set of $N$ parameters with $M>N$, no strict solutions, but only approximate ones, can be found since the problem has more equations or sets of model parameters that produce a given color than model parameters. Hence, the code finds the $N$-volume of approximate solutions by using $\chi^{2}$ minimization by the comparison with synthetic photometry. In the case of stellar clusters, the synthetic photometry is obtained from the average SED of population synthesis models. In these cases, and in addition to the success of the $10 \times$ LLL test, the $\chi^{2}$ minimization procedure is valid as long as (1) the synthetic photometry used has an intrinsic variance due to size of sample effects much lower than the uncertainty in the data (Cerviño \& Luridiana 2006), (2) the size of the sample effects do not produce biased synthetic photometry (Cerviño \& Valls-Gabaud 2003), and (3) the average values obtained from the models and used in the fit are representative of the intrinsic underlying distribution, that is, the underlying distribution of integrated luminosity has a Gaussian-like shape and the intrinsic variance can be translated to Gaussian-like confidence intervals, and $\chi^{2}$ minimization procedures are valid (Cerviño \& Luridiana $2006)^{11}$; this last requirement implies the analysis of highorder moments of the distribution and a first-order estimation of the mass and age of the cluster in the absence of external constraints and once the $10 \times$ LLL requirement is fulfilled by the observational data. However, from the LLL test we know

\footnotetext{
9 The latest version can be obtained from http://www.iaa.es/ jmaiz. 10 Synthesis models provide an average SED with a relative variance equal to zero only in the case of clusters with an infinite number of stars (Cerviño \& Luridiana 2006).

11 This requirement is actually linked with the $10 \times$ LLL and the unbiased photometry requirement.
} 


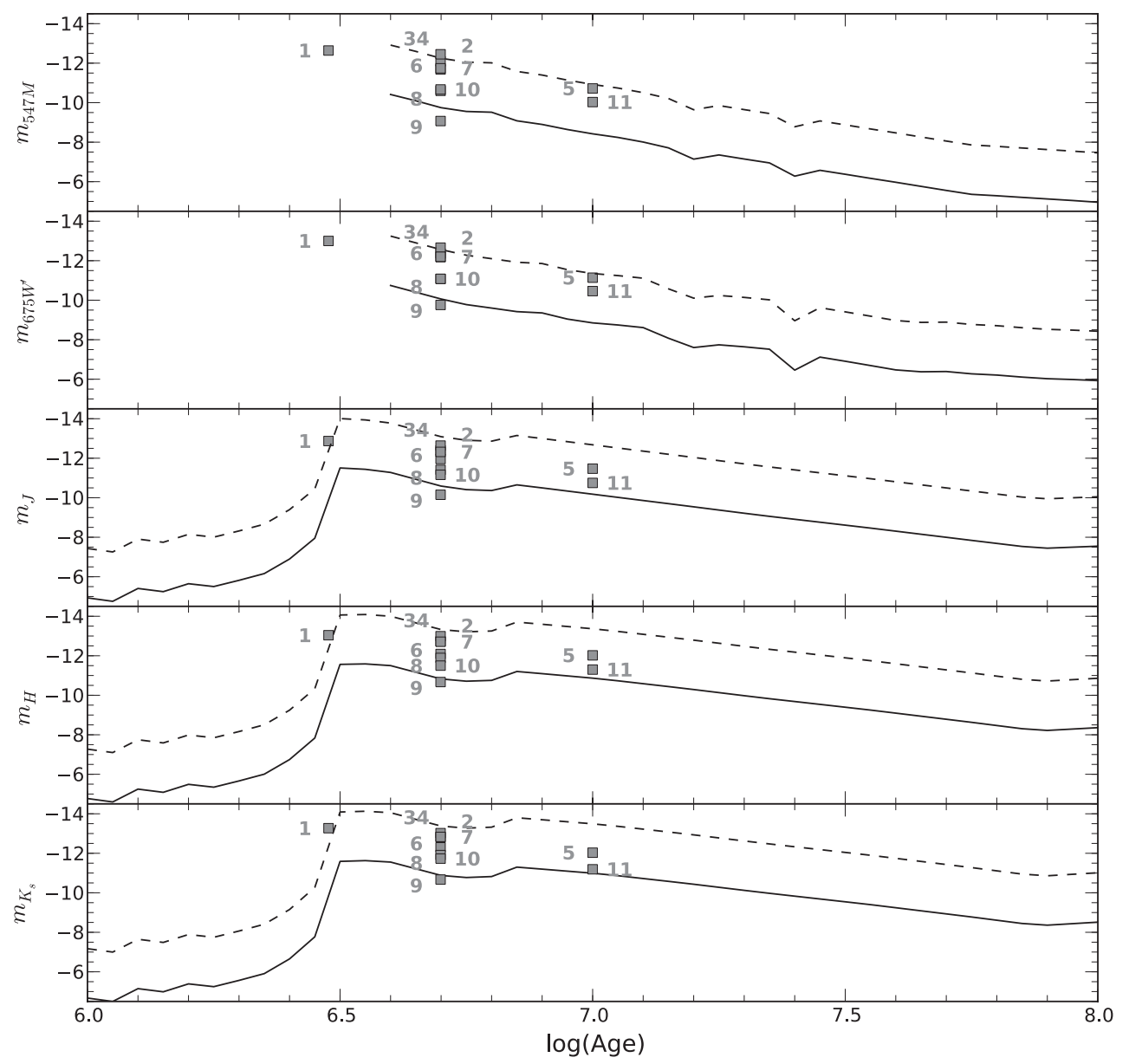

Figure 8. Lowest-luminosity limit for the optical and infrared filters used in the cluster photometry. Each cluster is located at the age resulting from the CHORIZOS analysis. The continuous line indicates de LLL and the dotted line the "safety" limit of LLL+2.5.

(A color version of this figure is available in the online journal.)

that the results from CHORIZOS should be interpreted with caution for the individual clusters analyzed (but it is valid for the cluster as a whole). We have used these results to obtain a first-order estimation of the effects of extinction and reddening which would modify the LLL test.

The first step of the program is to read the unreddened SED models, extinguish them according to a given extinction law, and obtain the synthetic photometry. To run the program, CHORIZOS requires a file with the photometry data and a series of input parameters, such as the model family, understood as the collection of SED from stellar atmosphere models or synthetic stellar populations, and the range of the parameters to be used. Then, CHORIZOS reads the model photometry from the tables calculated previously, interpolates, calculates the likelihood for each grid point, and writes the results for each of the four parameters.

In the case of our study, we have used the STARBURST99 models (Leitherer et al. 1999) with the implementation by Smith et al. (2002) which includes a grid of blanketed stellar atmospheres at different metallicities. We fixed two parameters - the known metallicity of NGC 5471 and the type of dust-leaving the amount of extinction and the age unconstrained.

Regarding extinction, CHORIZOS works with E(4405 $5495)$ and $R_{5495}$, which are the monochromatic equivalents to $E(B-V)$ and $R_{V}$, respectively, the values 4405 and 5495 referring to the central wavelength (in $\AA$ ) of the $B$ and $V$ filters. We used the standard type of dust characterized by a value $R_{5495}=3.1$. The metallicity of the region was assumed to be traced by the oxygen abundance. Recent derivations from detailed spectrophotometric studies give a mean value of $12+\log (\mathrm{O} / \mathrm{H})$ of $8.06 \pm 0.03$ for components $\mathrm{A}, \mathrm{C}$, and $\mathrm{D}$; a slightly lower abundance of $7.94 \pm 0.03$ is found for component B (Kennicutt et al. 2003). These values correspond to about 0.25 of the solar oxygen abundance, if the value by Asplund et al. (2005) for the solar oxygen abundance is assumed. Therefore, we used the value $Z=0.2 Z_{\odot}$ in CHORIZOS.

\section{DISCUSSION}

In this section, we discuss the results of the photometry in terms of the resolved stellar photometry in the CMD and in terms of the integrated properties, both of the individual clusters defined in Section 3.2 and of the whole star-forming complex. We attempt to bring all the results into a coherent picture of the past and present star formation processes in NGC 5471.

\subsection{Cluster Analysis}

Analyzing the integrated properties of a complex region like NGC 5471 is an exercise that has to be undertaken with care because the integrated photometry includes a wide range of regions with different cluster masses, ages, and extinction values. Thus, the result of synthesizing the integrated ultravioletto-NIR photometry with CHORIZOS, which finds the most likely single stellar population (SSP) under unconstrained inputs 
for mass, age, and extinction, is only of general interest as a guiding signpost. If we use only the optical-to-NIR photometry, CHORIZOS finds a most likely SSP with relatively high extinction and young age $3 \pm 2 \mathrm{Myr}$. If we include the FUV and NUV GALEX photometry then CHORIZOS finds two loci of maximum likelihood for a single SSP (one that corresponds to an age of $8 \pm 2 \mathrm{Myr}$ with moderate extinction and a second maximum at an age $60 \pm 15 \mathrm{Myr}$ and very low extinction). This result suggests that a significant fraction of the GALEX flux integrated within the 15 arcsec radius aperture from NGC 5471 is produced by a post-nebular stellar population of B stars (see also Waller et al. 1997).

Three previous studies have modeled the age of NGC 5471 using ultraviolet-to-optical spectra. Rosa \& Benvenuti (1994) use an HST/Faint Object Spectrograph (FOS) spectrum through an aperture of 4.3 arcsec around knot A. Mas-Hesse \& Kunth (1999) use an International Ultraviolet Explorer spectrum through an aperture of $10 \times 20$ arcsec and the FOS optical spectrum of Rosa \& Benvenuti (1994). Pellerin (2006) uses a FUSE spectrum through an aperture of $4 \times 20$ arcsec. All three works find that the UV spectrum of NGC 5471 can be explained with a young burst of less than about $5 \mathrm{Myr}$ and a relatively low extinction. These results are consistent with the detection of Wolf-Rayet stars in NGC 5471 (Mas-Hesse $\&$ Kunth 1999). However, these previous works may not be sensitive to an older population since they are based on the analysis of UV continuum and absorption lines ( $\mathrm{Si}$ IV and C IV) and optical emission lines as primary age indicators, and are thus biased toward younger knots with brighter ultraviolet fluxes while the integrated GALEX photometry through a large aperture is more sensitive to the extended, fainter, more evolved stellar population. ${ }^{12}$ Indeed, Meurer et al. (1995), Maoz et al. (1996), and Chandar et al. (2005) have found that the relative contribution from the cluster stellar population to the total UV flux in starbursts is only a fraction in the range $\sim 20 \%$ to $\sim 40 \%$, the majority of the UV flux coming from a diffuse fainter stellar population that is most prominent when the total flux is integrated, just as in the case of NGC 5471. In addition, the analysis performed with CHORIZOS focuses the results on the continuum shape. This discrepancy between ages inferred from emission lines and from the continuum points toward a complex SFH in NGC 5471 as we show below.

The integrated photometry analysis of the clusters identified in Section 3.2 may be affected by the size of sample effects due to the failure of the $10 \times$ LLL test in some of the clusters. However, we present here the analysis to show, as a case study, the possible interpretations. The values quoted must not be taken, in any case, as final values for cluster ages.

Figure 9 shows a color-color-H $\alpha$ plot of the individual clusters. The hypernova knot is not shown, and knots 3 and 4 have been combined into a single knot $34 .^{13}$ There is a clear correlation in the sense that redder knots have less $\mathrm{H} \alpha$ flux. The flux of $\mathrm{H} \alpha$ can decrease due to the aging of the burst or to different clusters having different masses, less massive bursts producing fewer ionizing photons. ${ }^{14}$ This latter could be the

\footnotetext{
12 Recall that the $1 \sigma$ detection for the GALEX data is $\mathrm{AB}_{\mathrm{FUV}}=$ 27.6 mag $\operatorname{arcsec}^{-2}$.

13 The nature of knots $3+4$ is not clear, it may be that both are part of the same cluster that has evolved sufficiently for the stellar winds to evacuate its center from ionized gas. Further higher spatial resolution IR and UV imaging is needed to establish more details.

14 Extinction may also play a small role but values significantly larger than reported in the literature, which are lower than $E(B-V)=0.14$, would be required.
}

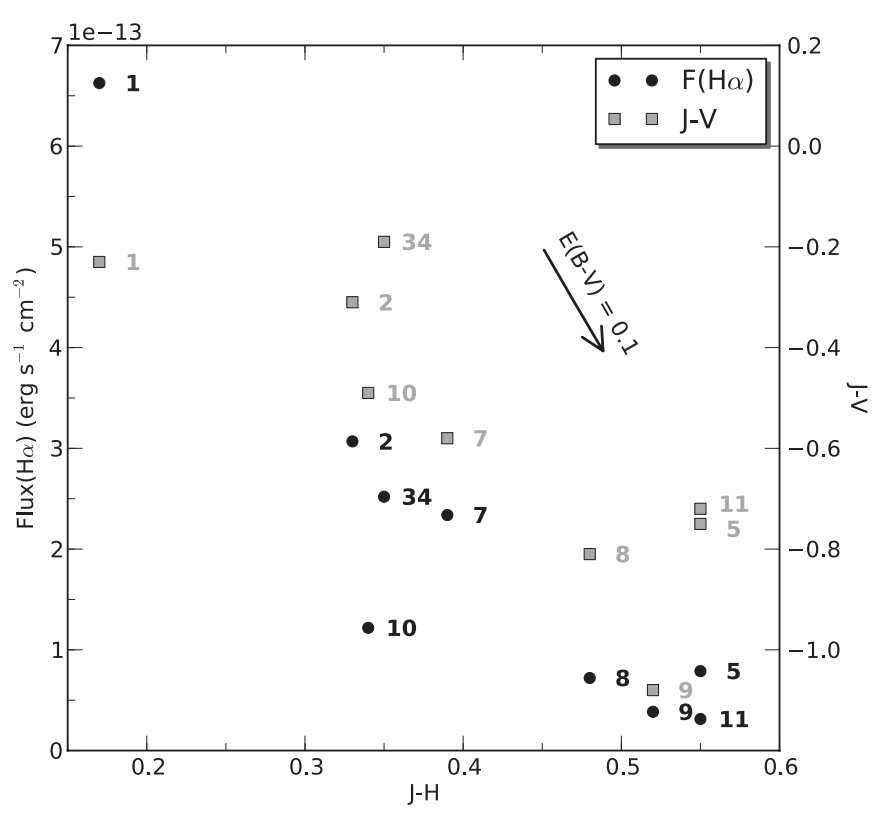

Figure 9. $\mathrm{H} \alpha$ and $J-V$ vs. $J-H$ plot for the clusters identified in Section 3.2 ( $V$ stands for the $547 \mathrm{M}$ filter). A clear correlation of decreasing $\mathrm{H} \alpha$ with redder colors points to an aging effect within the ongoing starburst phase.

(A color version of this figure is available in the online journal.)

case, including a relationship with color, when a strong size of sample effects are present and just a few stars are responsible for most of both the $\mathrm{H} \alpha$ and continuum emission (overluminous case) or by the absence of the most massive stars which mimics variations in the upper mass limit of the initial mass function (underluminous case; e.g., Villaverde et al. 2010).

Knot 1 is the brightest in $\mathrm{H} \alpha$, and the age estimations obtained by CHORIZOS give the youngest age, $3 \pm 2 \mathrm{Myr}$, with the lowest extinction. For knots 2, 3, 4, 7, 8, 9, 10, CHORIZOS estimates an intermediate age, $5 \pm 2 \mathrm{Myr}$, with an intermediate extinction, except may be knot 9 which has some indications for a somewhat higher extinction. Knots 5 and 11 have older age estimations, $9 \pm 3$ Myr. However, except for Knots 1, 2, and 34 , the age estimations can also be explained by size of sample effects with underluminous case. In these cases, age estimations, although apparently consistent, should not be considered to be final, pending a more complete analysis with additional data sets.

In summary, from the cluster analysis of individual bright knots and from the integrated photometry of the whole complex, there are clear indications that there is a complex star formation in the NGC 5471 event with indications of an older population of $\mathrm{B}$ stars and a luminous young ionizing cluster. This will be well established with the analysis of the CMD in the next section.

\subsubsection{Mass of the Ionized Gas}

The $\mathrm{H}^{+}$mass and the mass of the ionizing cluster may be derived from the $\mathrm{H} \alpha$ flux. The published values of internal extinction for NGC 5471 differ according to different authors, but they are lower than about $E(B-V)=0.14$ on average (Skillman 1985; Torres-Peimbert et al. 1989; Kennicutt \& Garnett 1996; Luridiana et al. 2002; Esteban et al. 2002). In NGC 604, Maíz Apellániz et al. (2004) have estimated that only about $10 \%$ of the $\mathrm{H} \alpha$ flux is missing in the higher opacity zones. Therefore, we have not attempted to correct the measured $\mathrm{H} \alpha$ flux to estimate other magnitudes from this value. The 
electron density assumed is $n_{e}=100 \mathrm{~cm}^{-3}$ (Luridiana et al. 2002).

The $\mathrm{H} \alpha$ flux in a $15^{\prime \prime}$ radius circular aperture (uncorrected for internal extinction) yields a lower limit to the luminosity $L_{\mathrm{H} \alpha} \sim 2.3 \times 10^{40} \mathrm{erg} \mathrm{s}^{-1}$. The total number of $\mathrm{H}$-ionizing photons, $Q\left(\mathrm{H}^{0}\right)$, estimated from Osterbrock (1989) is

$$
Q\left(\mathrm{H}^{0}\right)=\frac{\alpha_{\mathrm{B}}}{\alpha_{\mathrm{H} \alpha}^{\text {eff }}} \times \frac{L_{\mathrm{H} \alpha}}{h \nu_{\mathrm{H} \alpha}} \sim 2.2 \times 10^{52} \mathrm{~s}^{-1},
$$

where $\alpha_{\mathrm{B}} / \alpha_{\mathrm{H} \alpha}^{\text {eff }} \sim 2.99$, assuming case B recombination, $\alpha_{\mathrm{B}} \sim$ $2.59 \times 10^{-13} \mathrm{~cm}^{-3} \mathrm{~s}^{-1}$, and an effective recombination coefficient for $\mathrm{H} \alpha$ of $8.63 \times 10^{-14} \mathrm{~cm}^{-3} \mathrm{~s}^{-1}$ for $10^{4} \mathrm{~K}$, and $n_{e}=$ $100 \mathrm{~cm}^{-3}$ (Osterbrock 1989). Thus, the total (lower limit) ionized mass of the gas cloud is

$$
M_{\mathrm{H}^{+}}=Q\left(\mathrm{H}^{0}\right) \frac{m_{p}}{n_{e} \alpha_{\mathrm{B}}} \sim 7.3 \times 10^{5} M_{\odot} .
$$

From evolutionary models of single stellar population ionizing clusters and radiation-bounded $\mathrm{H}$ II regions, a relation between $Q\left(\mathrm{H}^{0}\right)$ per solar mass and the $\mathrm{H} \beta$ equivalent width can be found (Díaz 1998), which allows to estimate the stellar cluster mass by taking into account the cluster evolution:

$$
\log \left[Q\left(\mathrm{H}^{0}\right) / M_{\odot}\right]=0.86 \log [\mathrm{EW}(H \beta)]+44.48 .
$$

Hence, a lower limit for the mass of the cluster can be estimated by means of the $\mathrm{H} \beta$ equivalent width and the $\mathrm{H} \alpha$ luminosity of the region. Using the range of values $\operatorname{EW}(\mathrm{H} \beta)=$ 134-296 $\AA$ published by different authors (Luridiana et al. 2002; Esteban et al. 2002; Rosa \& Benvenuti 1994; Rosa 1981) and the value of $Q\left(\mathrm{H}^{0}\right)$ calculated above, Equation (5) yields a mass of the ionizing stellar cluster in the range $(0.6-1.1) \times 10^{6} M_{\odot}$. Using the calibration of Kennicutt (1998) and the luminosity that we measure, $L_{\mathrm{H} \alpha} \sim 2.3 \times 10^{40} \mathrm{erg} \mathrm{s}^{-1}$, we obtain a star formation rate (SFR) of $0.18 M_{\odot} \mathrm{yr}^{-1}$ or $2.1 \times$ $10^{-7} M_{\odot} \mathrm{yr}^{-1} \mathrm{pc}^{-2}$ (taking the area of the region as the circular aperture defined in Figure 4), that translates into a cluster mass of $10^{6} M_{\odot}$ for a burst duration of $10 \mathrm{Myr}$, which is the mean duration of the current burst implied by the analysis in the previous section. Nevertheless, it is known that this calibration was computed for a particular star formation episode at nearly constant rate during more than $100 \mathrm{Myr}$, so it might not be suitable for bursty systems. We have applied the new calibration by Otí-Floranes \& Mas-Hesse (2010), ${ }^{15}$ which makes a distinction between instantaneous (IB) and extended burst (EB). We used our measured $\mathrm{H} \alpha$ luminosity, no color excess, and a Salpeter initial mass function (IMF) in the range $1-100 M_{\odot}$ as input. For the case of instantaneous burst, Otí-Floranes \& Mas-Hesse (2010) use the concept of star formation strength (SFS), defined as the total mass of gas transformed into stars during the burst, giving three different ages for the IB case. We obtained an SFS of $1.5 \times 10^{6} M_{\odot}$ for a burst of $4 \mathrm{Myr}, 2.3 \times 10^{6} M_{\odot}$ for $5 \mathrm{Myr}$, and $1.0 \times 10^{7} M_{\odot}$ for the $6 \mathrm{Myr}$ case. For an EB, the results are given in terms of the standard SFR, with three different tabulated ages, namely, 10, 30, and $250 \mathrm{Myr}$. However, in this age range, the results are independent of the age for the $\mathrm{H} \alpha$ estimator. If the region would be experiencing a continuous constant star formation, the SFR given by this calibration would be

\footnotetext{
15 Their web tool can be found at

http://www.laeff.cab.inta-csic.es/research/sfr/.
}

$0.1 M_{\odot} \mathrm{yr}^{-1}$, less than a factor of two lower than the calibration by Kennicutt (1998). ${ }^{16}$

Thus, we find that the masses of ionized gas and of the stellar cluster in the current star formation event are both of the same order of magnitude, close to $10^{6} M_{\odot}$. This similarity may be understood in terms of a very high efficiency in star formation. Given that this is not the first event of star formation in NGC 5471 in the last $100 \mathrm{Myr}$, this high star formation efficiency takes on an important meaning that implies that there has to be a large reservoir of gas to maintain this important star formation activity.

Although optical images show NGC 5471 apparently isolated in the outskirts of M101, the H I distribution of M101 shows that NGC 5471 is, in fact, one of the brightest regions in $\mathrm{HI}$, located in a spiral arm (cf. Figure 1). The thermal radio continuum emission from NGC 5471 has the highest observed central emission measure of all the M101 complexes. Closely coincident with this $\mathrm{H}$ II radio continuum peak, there is an $\mathrm{H}_{\mathrm{I}}$ concentration of nearly $10^{8} M_{\odot}$ (Viallefond et al. 1981). The general distribution of the H I gas in M101 follows that of the diffuse FUV emission, with enhancements in the $21 \mathrm{~cm}$ line emission seen near FUV peaks. Smith et al. (2000) include NGC 5471 as one of their 35 photodissociation region candidates. Their Ultraviolet Imaging Telescope FUV flux measurement is in good agreement with our GALEX measurement of $9.8 \times 10^{-14} \mathrm{erg} \mathrm{cm}^{-2} \mathrm{~s}^{-1} \AA^{-1}$, and NGC 5471 is clearly the brightest FUV peak in the whole M101 galaxy. This is reflected in a decreased molecular content surrounding NGC 5471 which has been dissociated by the high star formation efficiency, the highest in M101 as calculated by Giannakopoulou-Creighton et al. (1999).

\section{2. $C M D$}

The photometry of the individual stars presented in the CMD, $m_{547 \mathrm{M}}-m_{675 \mathrm{~W}^{\prime}}(V-R)$ versus $m_{547 \mathrm{M}}(V)$, of Figure 10 is a powerful tool for further understanding the SFH in NGC 5471. The isochrones represented in the figure have been divided into five age bins (4-10, 10-25, 25-50, 50-75, 75-100) Myr and drawn by their concave hull, shown with different colors. The right-hand side of the figure shows the luminosity function, which peaks at $M_{547 \mathrm{M}}=24$, and becomes incomplete for fainter stars, as indicated by the $50 \%$ completeness limit shown. We recall that this CMD is the result of a careful process of calibration, measurement, and cleaning, as detailed in Section 3.1 above. We have not included in the CMD those point-like sources with a partially resolved PSF, that are likely to be compact clusters. At the distance of M101 some of the point sources are unresolved multiple systems, some of which may have been included in the CMD, but these will not really affect any of the arguments that we present here.

A glance at the CMDs tells us at once that star formation has been proceeding more or less continuously for the last 100 Myr. The diagram is populated by stars of $\sim 4-50 M_{\odot}$. We can readily identify clear structures in the diagram. There is a welldefined main-sequence at $V-R \sim-0.1$, which begins with the brightest star at 19 mag (that corresponds to an age of $\sim 4 \mathrm{Myr}$ ) and goes all the way down to $25 \mathrm{mag}$. Red stars span a range of 3-4 mag in brightness, indicating that star formation has occurred during the last $\sim 15-70$ Myr. There are some traces of the blue plume at $M_{V}=-9$, indicating ongoing star formation.

16 This calibration integrates a Salpeter IMF in the mass range $0.1-100 M_{\odot}$, which predicts a larger mass. 


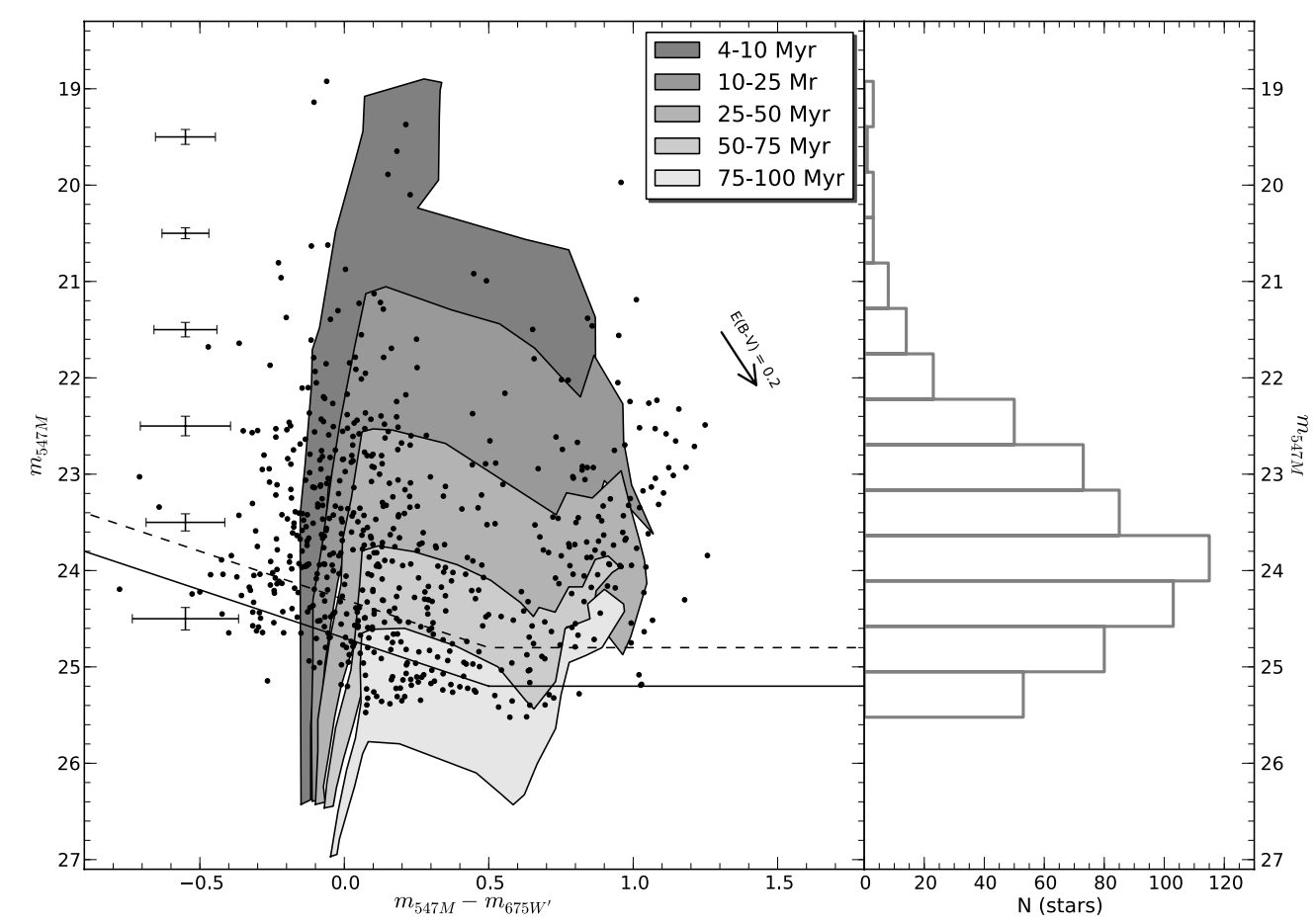

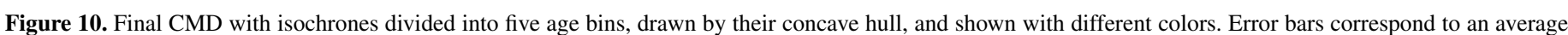

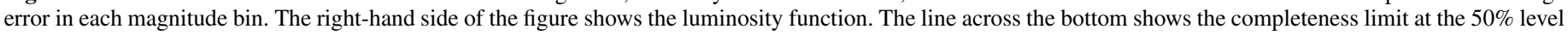
in the core (dashed line) and in the rest of the image (solid line).

(A color version of this figure is available in the online journal.)

A strip of stars following a patch from [0.6, 25] to [1.1, 22.5] form the red giant branch.

\subsubsection{Mass in Stars and Star Formation History}

In order to quantify the relative importance of the present burst to the SFH during the previous 100 Myr in NGC 5471, we make a rough estimate of the stellar mass from the CMD. We follow three different approaches. One method consists of assigning a mass to each of the stars in the CMD, corresponding to the most probable isochrone. This is done considering a 2D Gaussian probability density distribution centered on the distance in the color-magnitude space of a given observed star to the isochrones with dispersions being the estimated errors for the photometry. Once a mass has been assigned to a star, we count the stars in the mass range 6-55 $M_{\odot}$ and integrate a Salpeter IMF in the mass range $1-100 M_{\odot}$. The result of this calculation gives $\sim 2 \times 10^{5} M_{\odot}$. The second method is as follows: first, we assign a mean stellar mass of $6 M_{\odot}$ to the stars in the most populated bin of the luminosity function, $m_{547 \mathrm{M}}=23.5$. This mass is then scaled up by the ratio of the total $m_{547 \mathrm{M}}$ of NGC 5471 to the magnitude of the bin $m_{547 \mathrm{M}}=23.5$. The resulting mass is used to normalize the IMF at the bin corresponding to $6 M_{\odot}$. The result of integrating a Salpeter IMF in the range $1-100 M_{\odot}$ thus normalized gives a value of $\sim 5 \times 10^{5} M_{\odot}$.

A third independent method for estimating the mass is based on the fit of the CMD by means of Hess diagrams. We have used the code STARFISH ${ }^{17}$ (Harris \& Zaritsky 2001, 2002), with the latest patch sets, to find a best fit to the CMD by a linear combination of Hess diagrams at different ages. We have used our own set of isochrones described in Section 3.1.3. The best-fit SFH of NGC 5471 is shown in Figure 11. Figure 12 shows the synthetic CMD of NGC 5471 constructed using the

\footnotetext{
17 Available at http://www.noao.edu/staff/jharris/SFH/.
}

SFH amplitudes output of the best-fit SFH; the observed CMD is also shown for reference. The synthetic main sequence seems tighter and bluer compared to the observed one, probably due to differential reddening and underestimated errors. The SFH derived seems to be consistent with a continuous one, but the errors only allow to take into consideration two episodes: a dominant young burst $<20 \mathrm{Myr}$, preceded by a longer event during the period 20-100 Myr. According to the SFR, the integrated mass in stars of the youngest event is comparable to the oldest one, with $\sim 10^{6} M_{\odot}$.

Although these methods give only an order of magnitude estimate, we can conclude that the integrated mass of stars formed in the past 20-100 Myr is of the order of magnitude of the current star formation event, that we have calculated above as $\sim 10^{6} M_{\odot}$. If the star formation proceeds in an isolated gas cloud, we would expect that the rate at which stars form would decrease with time, as less gas is left available to form new stars. This is what is observed in many giant $\mathrm{H}$ II regions with two or more generations of star formation, where the latest event is less massive than the previous. In the case of NGC 5471, the results from the integrated photometry and from the CMD indicate that the current star-forming event is as massive as those during the previous 20-100 Myr. We can understand this in the context of the large Hi spiral arm in which the region is immersed (cf. Section 4.1.1). In this respect, Waller et al. (1997) conclude from a morphological study of M101 that NGC 5471 may be the result of tidal interactions of M101 with the nearby galaxies NGC 5477 and NGC 5474 in the time interval 100-1000 Myr ago.

\subsubsection{Spatial Distribution of the Star Formation History}

Given the long duration of the star formation in NGC 5471, $\sim 100 \mathrm{Myr}$, and its large size $\sim 1 \mathrm{kpc}$, it is natural to question 


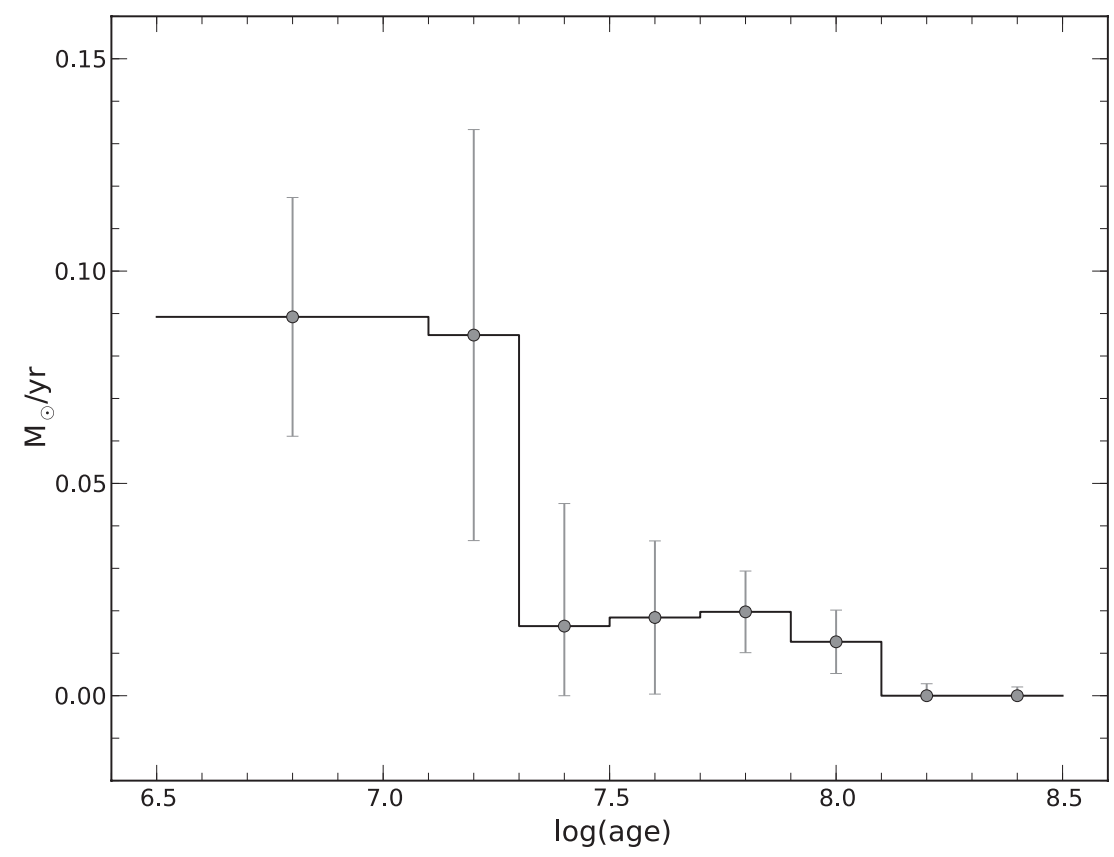

Figure 11. STARFISH recovered star formation history of NGC 5471.

(A color version of this figure is available in the online journal.)
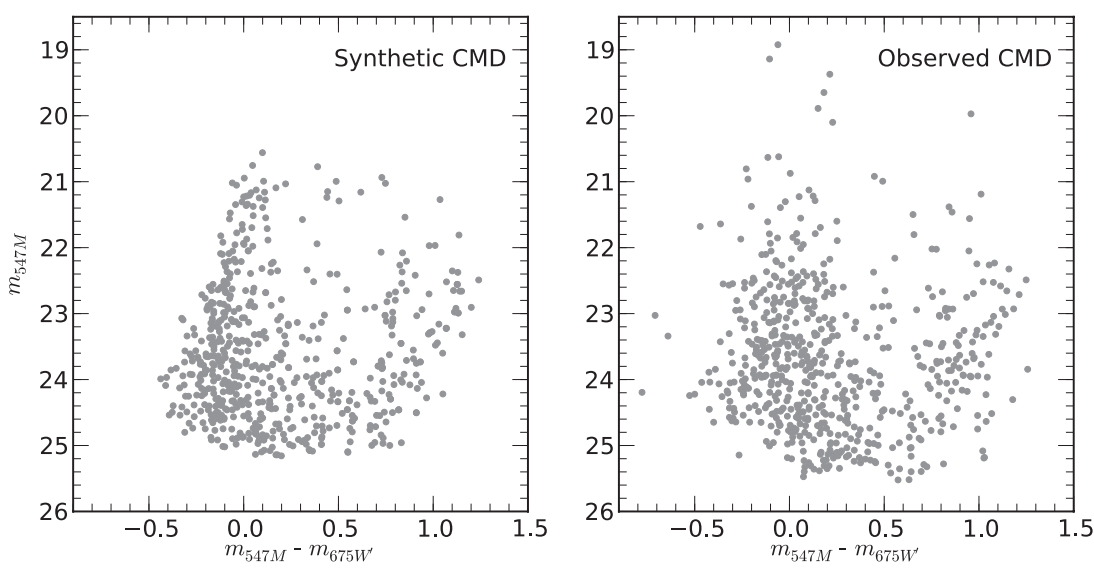

Figure 12. NGC 5471 synthetic CMD based on the best-fit SFH solution of STARFISH (left) and the observed one (right).

(A color version of this figure is available in the online journal.)

the spatial location of the star formation through time along the extent of the region. In order to visualize this, we have selected four different areas in the CMD and spatially located the stars in these four boxes. Figure 13 displays the resulting distributions. The youngest and more massive stars (top left panel) are clearly concentrated in the main star-forming emission-line knots, implying that massive stars are now forming mainly in clusters. Only two stars are not directly associated with these knots, one of which belongs to an isochrone of $20 \mathrm{Myr}$. The bottom right panel displays the distribution of intermediate mass stars $\left(\sim 5-10 M_{\odot}\right)$ older than about $50 \mathrm{Myr}$; these stars are distributed mainly toward the halo, with only a few in the core of the region. The top right and bottom left panels show the distribution of intermediate-mass stars $\left(\sim 10-15 M_{\odot}\right)$, both those which are in the main sequence and those which have already evolved out of it. The distributions of stars in these two CMD boxes are clearly concentrated fairly uniformly throughout the core of NGC 5471; some of the stars in these two boxes are found in the halo, with a sparse distribution somewhat similar to the older lower mass stars in the bottom right panel. Because the stars in these two intermediate boxes are of the same mass but they are found in a range of evolutionary stages, this implies that star formation has proceeded more or less uniformly in the core for the last 20-50 Myr.

From this analysis of the spatial distribution of the CMD, a clear picture emerges in which the star formation in the complex NGC 5471 has proceeded in a general spatio-temporal sequence from the periphery to the core. During the first epoch, 50$100 \mathrm{Myr}$ ago, the star formation occurred along the periphery and the core, then a second major event $\sim 10-20$ Myr ago was more or less uniformly distributed mainly in the core, and finally the current ionizing star-forming event is mostly concentrated in the singular bright clusters located inside the core, where the gas reservoir is presumably higher. ${ }^{18}$ At the same time, we have seen from both the integrated photometry analysis and the CMD, that as the events proceeded concentrating from the periphery to the core clusters, the mass of gas formed into stars seems to be

\footnotetext{
18 Note that the age dating results from the individual cluster photometry is compatible with this age-spatial distribution of the younger stars in the CMD, and this reinforces the age results obtained for the clusters.
} 

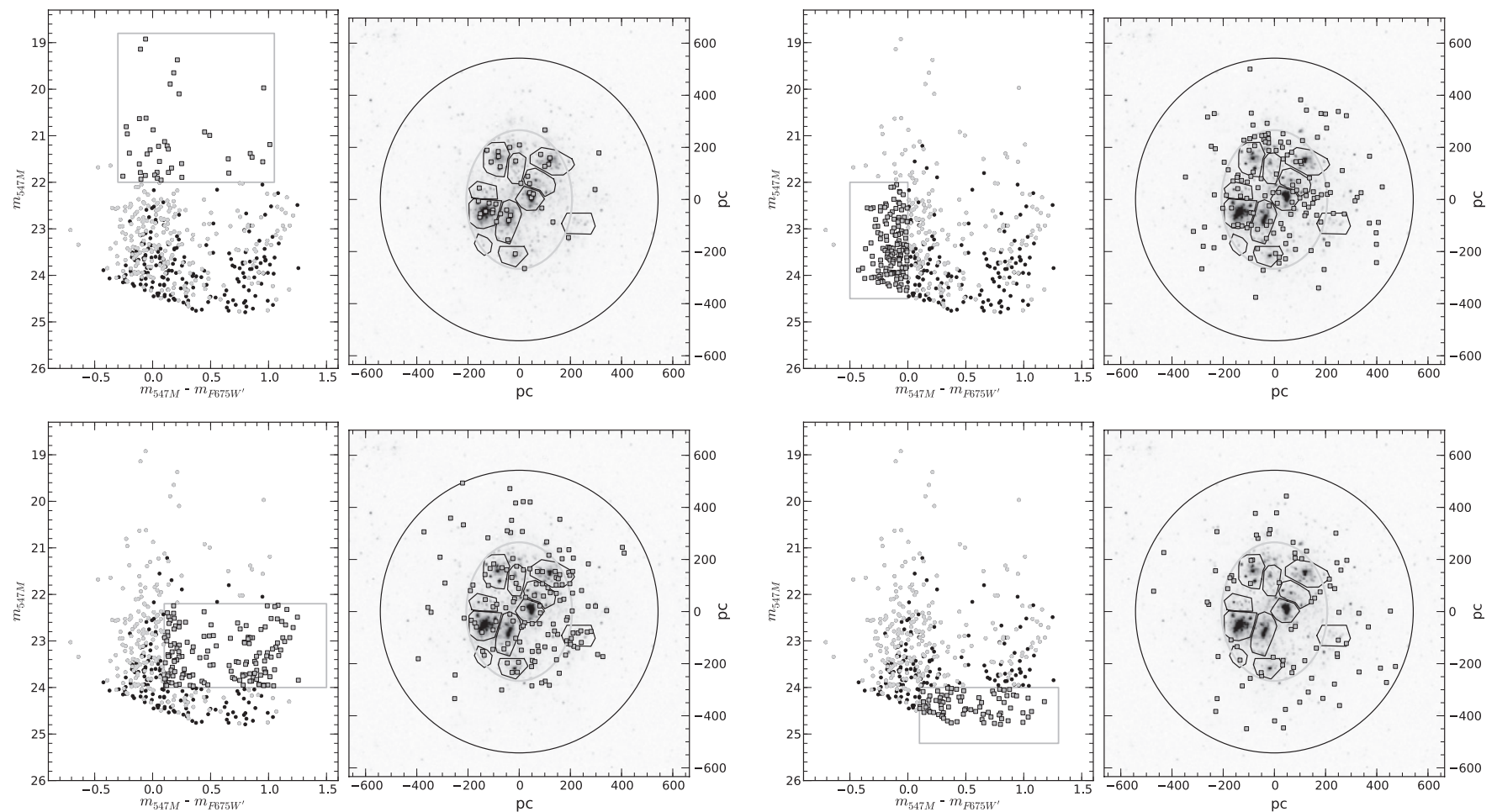

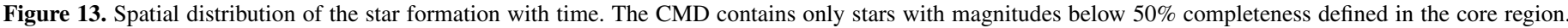

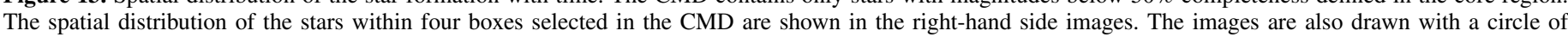

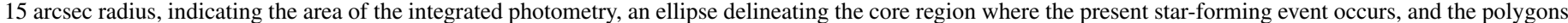

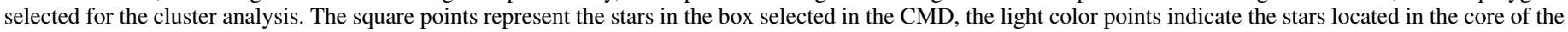

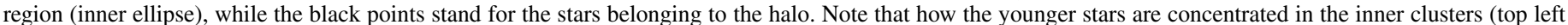
panel) and intermediate and older stars populate uniformly the region.

(A color version of this figure is available in the online journal.)

of the same order of magnitude, but more concentrated in time, with higher star formation rate.

We note that the current event of ionizing clusters is apparently contained within a large bubble which defines the core of NGC 5471. This bubble, of projected size $400 \times 550 \mathrm{pc}$, is clearly defined geometrically (cf. Figure 2) and kinematically (Muñoz-Tuñón et al. 1995) and is likely to have been produced by the stars that formed $\sim 20$ Myr ago. ${ }^{19}$

There are other examples that seem to follow the general process of star formation that we have outlined here for NGC 5471. Úbeda et al. (2007) find an inward sequence of star formation and a bubble for cluster I-A in NGC 4214. Walborn \& Blades (1997) find a triggered spatio-temporal distribution of stars in 30 Doradus, but this sequence occurs at the scale of the individual clusters in NGC 5471. Indeed there are indications that at this smaller scale, $\sim 50 \mathrm{pc}$, the evolution of the different individual clusters indicated by the age dating is also reflected in their morphology (e.g., as mentioned in the case of 3+4), but a detailed study at these scales requires the high-resolution UV-optical-IR imaging that can only be provided by the WF3 camera on board HST.

\section{CONCLUSIONS}

We have performed a photometric study of NGC 5471, a GEHR in the outskirts of the spiral galaxy M101, to derive its SFH by means of resolved stellar photometry and integrated cluster photometry. Furthermore, integrated photometry of the 19 The results from a kinematical analysis of the expansion of this bubble are
in progress and will be published elsewhere. whole region, using data from GALEX (ultraviolet), HST/ WFPC2 (optical), and TNG (near-infrared), yields two possible solutions for a single stellar population: one that corresponds to an age of $\sim 8 \mathrm{Myr}$ with moderate extinction and one with an age around $60 \mathrm{Myr}$ and very low extinction. This degeneration is typical of this kind of approach when spatially integrated data are used and reflects the complexity of GEHRs that include clusters with different ages and extinction values.

From the photometric analysis of the 11 clusters defined on the IR H image, a correlation emerges in the sense that redder knots have less $\mathrm{H} \alpha$ flux, showing a clear aging trend. The ages range from $3 \mathrm{Myr}$ for the youngest cluster up to $10 \mathrm{Myr}$ for the oldest one. However, the lowest-luminosity limit test prevents us from taking these estimates at face value, since only a few individual clusters satisfy the required limit.

The complex history of star formation of NGC 5471 revealed by the cluster analysis is confirmed by the resolved stellar analysis. From the CMD it is clear that star formation has been proceeding continuously with more or less intensity for the last $100 \mathrm{Myr}$. The well-defined main sequence gives a youngest age of about $4 \mathrm{Myr}$, while red stars, which span a range of 3-4 mag, indicate that star formation has occurred during the last 15-70 Myr.

We have found that the masses of the ionized gas and of the stellar cluster in the current star formation event are both of the same order of magnitude, around $10^{6} M_{\odot}$, which may be understood in terms of a very high efficiency. In fact, the SFS of NGC 5471 is more than a factor of two that of NGC 604 in M33 (Relaño \& Kennicutt 2009). This implies that there has to be a large reservoir of gas to maintain this star formation rate, 
something that is confirmed by the huge $\mathrm{H}_{\mathrm{I}}$ concentration in which the region is located.

From the spatial distribution of the stars, we can conclude that the star formation in NGC 5471 has proceeded in a general spatio-temporal sequence which was more extended in the past and now is concentrated in clusters in the core. The current event of ionizing clusters is contained within a large bubble, which is likely to have been produced by the stars that formed $\sim 20 \mathrm{Myr}$ ago. This leads to the general question of whether a low density "seed" generation of evolved intermediate-mass stars is in fact a prerequisite to trigger a starburst event (Brandner 2002). More UV-optical-IR data at higher spatial resolution, such as that which can be provided by the WF3 camera on board HST, are obviously needed in order to have a conclusive answer.

We have benefited from comments and suggestions from Emilio Alfaro, David Martínez Delgado, and Nolan Walborn. This work has been funded by DGICYT programs AYA2004-02703, AYA2007-64712, AYA2004-08260-C03-03, and AYA2007-67965-C03-03. R.G.B. acknowledges support from the Spanish MEC through FPI grant BES-2005-6910 and from the China National Postdoc Fund Grant No. 20100480144. This work has made use of data from the archives of the NASA/ ESA Hubble Space Telescope, obtained at the Space Telescope Science Institute, which is operated by the Association of Universities for Research in Astronomy, Inc., under NASA contract NAS5-26555, and of data from the Telescopio Nazionale Galileo, operated on the island of La Palma by the Galileo Galilei Foundation INAF in the Spanish Observatorio del Roque de Los Muchachos of the Instituto de Astrofísica de Canarias. Thanks to cheerful Francesca Ghinassi for her help during the TNG observations. We thank an anonymous referee for very useful comments that improved the presentation of the paper.

\section{REFERENCES}

Anderson, J., \& King, I. R. 1999, PASP, 111, 1095

Aparicio, A., \& Gallart, C. 1995, AJ, 110, 2105

Asplund, M., Grevesse, N., \& Sauval, A. J. 2005, in ASP Conf. Ser. 336, Cosmic Abundances as Records of Stellar Evolution and Nucleosynthesis, ed. T. G. Barnes, III \& F. N. Bash (San Francisco, CA: ASP), 25

Bellazzini, M., Ferraro, F. R., \& Buonanno, R. 1999, MNRAS, 307, 619

Bertelli, G., Bressan, A., Chiosi, C., Fagotto, F., \& Nasi, E. 1994, A\&AS, 106, 275

Bianchi, L., et al. 2005, ApJ, 619, L71

Brandner, W. 2002, in ASP Conf. Ser. 285, Modes of Star Formation and the Origin of Field Populations, ed. E. K. Grebel \& W. Brandner (San Francisco, CA: ASP), 105

Braun, R. 1995, A\&AS, 114, 409

Cardelli, J. A., Clayton, G. C., \& Mathis, J. S. 1989, ApJ, 345, 245

Cerviño, M., \& Luridiana, V. 2004, A\&A, 413, 145

Cerviño, M., \& Luridiana, V. 2006, A\&A, 451, 475

Cerviño, M., \& Valls-Gabaud, D. 2003, MNRAS, 338, 481

Chandar, R., Leitherer, C., Tremonti, C. A., Calzetti, D., Aloisi, A., Meurer, G. R., \& de Mello, D. 2005, ApJ, 628, 210

Chen, C.-H. R., Chu, Y.-H., Gruendl, R., Lai, S.-P., \& Wang, Q. D. 2002, AJ, 123,2462

Díaz, Á. I. 1998, Ap\&SS, 263, 143

Dolphin, A. E. 2000a, PASP, 112, 1383
Dolphin, A. E. 2000b, PASP, 112, 1397

Esteban, C., Peimbert, M., Torres-Peimbert, S., \& Rodríguez, M. 2002, ApJ, 581,241

Giannakopoulou-Creighton, J., Fich, M., \& Wilson, C. D. 1999, ApJ, 522, 238

Girardi, L., \& Bertelli, G. 1998, MNRAS, 300, 533

Girardi, L., Bertelli, G., Bressan, A., Chiosi, C., Groenewegen, M. A. T., Marigo, P., Salasnich, B., \& Weiss, A. 2002, A\&A, 391, 195

Girardi, L., Bressan, A., Bertelli, G., \& Chiosi, C. 2000, A\&AS, 141, 371

Harris, J., \& Zaritsky, D. 2001, ApJS, 136, 25

Harris, J., \& Zaritsky, D. 2002, in ASP Conf. Ser. 274, Observed HR Diagrams and Stellar Evolution, ed. T. Lejeune \& J. Fernandes (San Francisco, CA: ASP), 600

Heyer, I., \& Biretta, J. A. 2004, WFPC2 Instrument Handbook, Version 9.0 (Baltimore, MD: STScI)

Holtzman, J., et al. 1995, PASP, 107, 156

Jenkins, L. P., Roberts, T. P., Warwick, R. S., Kilgard, R. E., \& Ward, M. J. 2004, MNRAS, 349, 404

Kennicutt, R. C., Jr. 1998, ApJ, 498, 541

Kennicutt, R. C., Jr., Bresolin, F., \& Garnett, D. R. 2003, ApJ, 591, 801

Kennicutt, R. C., Jr., \& Garnett, D. R. 1996, ApJ, 456, 504

Krist, J. 1995, in ASP Conf. Ser. 77, Astronomical Data Analysis Software and Systems IV, ed. R. A. Shaw, H. E. Payne, \& J. J. E. Hayes (San Francisco, CA: ASP), 349

Leitherer, C., et al. 1999, ApJS, 123, 3

Luridiana, V., Esteban, C., Peimbert, M., \& Peimbert, A. 2002, RevMexAA, 38, 97

Luridiana, V., Pérez, E., \& Cerviño, M. 2003, AJ, 125, 3196

Maíz Apellániz, J. 2001, ApJ, 563, 151

Maíz Apellániz, J. 2004, PASP, 116, 859

Maíz Apellániz, J. 2007, in ASP Conf. Ser. 364, The Future of Photometric, Spectrophotometric and Polarimetric Standardization, ed. C. Sterken (San Francisco, CA: ASP), 227

Maíz Apellániz, J., Pérez, E., \& Mas-Hesse, J. M. 2004, AJ, 128, 1196

Maoz, D., Barth, A. J., Sternberg, A., Filippenko, A. V., Ho, L. C., Macchetto, F. D., Rix, H.-W., \& Schneider, D. P. 1996, AJ, 111, 2248

Martin, D. C., et al. 2005, ApJ, 619, L1

Mas-Hesse, J. M., \& Kunth, D. 1999, A\&A, 349, 765

Meurer, G. R., Heckman, T. M., Leitherer, C., Kinney, A., Robert, C., \& Garnett, D. R. 1995, AJ, 110, 2665

Mighell, K. J., Rich, R. M., Shara, M., \& Fall, S. M. 1996, AJ, 111, 2314

Morrissey, P., et al. 2005, ApJ, 619, L7

Muñoz-Tuñón, C., Gavryusev, V., \& Castañeda, H. O. 1995, AJ, 110, 1630

Osterbrock, D. 1989, Astrophysics of Gaseous Nebulae and Active Galactic Nuclei (Mill Valley, CA: Univ. Science Book)

Otí-Floranes, H., \& Mas-Hesse, J. M. 2010, A\&A, 511, A61

Pellerin, A. 2006, AJ, 131, 849

Relaño, M., \& Kennicutt, R. C. 2009, ApJ, 699, 1125

Rosa, M. 1981, PhD thesis, Landessternwarte Heidelberg/Königstuhl

Rosa, M. R., \& Benvenuti, P. 1994, A\&A, 291, 1

Schlegel, D. J., Finkbeiner, D. P., \& Davis, M. 1998, ApJ, 500, 525

Shaklan, S., Sharman, M. C., \& Pravdo, S. H. 1995, Appl. Opt., 34, 6672

Skillman, E. D. 1985, ApJ, 290, 449

Smith, D. A., Allen, R. J., Bohlin, R. C., Nicholson, N., \& Stecher, T. P. 2000, ApJ, 538, 608

Smith, L. J., Norris, R. P. F., \& Crowther, P. A. 2002, MNRAS, 337, 1309

Stetson, P. B., \& Harris, W. E. 1988, AJ, 96, 909

Stetson, P. B., et al. 1998, ApJ, 508, 491

Torres-Peimbert, S., Peimbert, M., \& Fierro, J. 1989, ApJ, 345, 186

Úbeda, L., Maíz Apellániz, J., \& MacKenty, J. W. 2007, AJ, 133, 932

Viallefond, F., Allen, R. J., \& Goss, W. M. 1981, A\&A, 104, 127

Villaverde, M., Cerviño, M., \& Luridiana, V. 2010, A\&A, 522, A49

Walborn, N. R., Barbá, R. H., Brandner, W., Rubio, M., Grebel, E. K., \& Probst, R. G. 1999, AJ, 117, 225

Walborn, N. R., \& Blades, J. C. 1997, ApJS, 112, 457

Waller, W. H., et al. 1997, ApJ, 481, 169

Westera, P., Lejeune, T., Buser, R., Cuisinier, F., \& Bruzual, G. 2002, A\&A, 381,524 